\title{
CAUGHT IN THE ACT: GAS AND STELLAR VELOCITY DISPERSIONS IN A FAST QUENCHING COMPACT STAR-FORMING GALAXY AT $z \sim 1.7$
}

\author{
Guillermo Barro $^{1}$, Sandra M. Faber ${ }^{1}$, Avishai Dekel ${ }^{2}$, Camilla Pacifici $^{3}$, Pablo G. Pérez-González ${ }^{4}$, Elisa Toloba ${ }^{1}$, \\ David C. Koo ${ }^{1}$, Jonathan R. Trump ${ }^{5,13}$, Shigeki Inoue ${ }^{2}$, Yicheng Guo ${ }^{1}$, Fengshan Liu ${ }^{6}$, Joel R. Primack ${ }^{7}$, \\ Anton M. Koekemoer ${ }^{8}$, Gabriel Brammer ${ }^{8}$, Antonio Cava ${ }^{9}$, Nicolas Cardiel ${ }^{4}$, Daniel Ceverino ${ }^{10}$, Carmen Eliche $^{4}$, \\ Jerome J. Fang ${ }^{1}$, Steven L. Finkelstein ${ }^{11}$, Dale D. Kocevski ${ }^{12}$, Rachael C. Livermore ${ }^{11}$, and Elizabeth McGrath ${ }^{12}$ \\ ${ }^{1}$ University of California, Santa Cruz, USA \\ 2 The Hebrew University, Israel \\ ${ }^{3}$ Yonsei University Observatory, South Korea \\ ${ }^{4}$ Universidad Complutense de Madrid, Spain \\ ${ }^{5}$ Pennsylvania State University, USA \\ ${ }^{6}$ Shenyang Normal University, China \\ ${ }^{7}$ Santa Cruz Institute for Particle Physics, USA \\ ${ }^{8}$ Space Telescope Science Institute, USA \\ ${ }^{9}$ Observatoire de Geneve, Switzerland \\ ${ }^{10}$ Centro de Astrobiologia, CSIC-INTA, Spain \\ ${ }^{11}$ The University of Texas at Austin, USA \\ ${ }^{12}$ Colby College, USA \\ Received 2015 March 27; accepted 2016 February 16; published 2016 March 29
}

\begin{abstract}
We present Keck I MOSFIRE spectroscopy in the $Y$ and $H$ bands of GDN-8231, a massive, compact, star-forming galaxy at a redshift of $z \sim 1$.7. Its spectrum reveals both $\mathrm{H} \alpha$ and [N $\mathrm{II}]$ emission lines and strong Balmer absorption lines. The $\mathrm{H} \alpha$ and Spitzer MIPS $24 \mu \mathrm{m}$ fluxes are both weak, thus indicating a low star-formation rate of SFR $\lesssim 5-10 M_{\odot} \mathrm{yr}^{-1}$. This, added to a relatively young age of $\sim 700 \mathrm{Myr}$ measured from the absorption lines, provides the first direct evidence for a distant galaxy being caught in the act of rapidly shutting down its star formation. Such quenching allows GDN-8231 to become a compact, quiescent galaxy, similar to three other galaxies in our sample, by $z \sim 1.5$. Moreover, the color profile of GDN-8231 shows a bluer center, consistent with the predictions of recent simulations for an early phase of inside-out quenching. Its line-of-sight velocity dispersion for the gas, $\sigma_{\text {LOS }}^{\text {gas }}=127 \pm 32 \mathrm{~km} \mathrm{~s}^{-1}$, is nearly $40 \%$ smaller than that of its stars, $\sigma_{\text {Los }}^{\star}=215 \pm 35 \mathrm{~km} \mathrm{~s}^{-1}$. Highresolution hydro-simulations of galaxies explain such apparently colder gas kinematics of up to a factor of $\sim 1.5$ with rotating disks being viewed at different inclinations and/or centrally concentrated star-forming regions. A clear prediction is that their compact, quiescent descendants preserve some remnant rotation from their starforming progenitors.
\end{abstract}

Key words: galaxies: high-redshift - galaxies: photometry

\section{INTRODUCTION}

The formation and structural evolution of the first quiescent galaxies at $z \gtrsim 2$ have been the subject of considerable discussion in recent years. Since the first papers reporting their remarkably compact nature compared to quiescent galaxies of similar stellar mass at low redshift, many studies have verified their small sizes and characterized its growth with cosmic time (Daddi et al. 2005; Trujillo et al. 2007; Buitrago et al. 2008; Cimatti et al. 2008; van Dokkum et al. 2008; Damjanov et al. 2009; Cassata et al. 2011; Newman et al. 2012a; Szomoru et al. 2012; Carollo et al. 2013; van der Wel et al. 2014). However, the mechanisms responsible for the formation of such compact objects are still unclear.

A crucial step forward in understanding these formation processes is the recent discovery of a population of massive $\left(\log \left(M / M_{\odot}\right)>10\right)$, compact, star-forming galaxies (SFGs) at $z \gtrsim 2$ (Wuyts et al. 2011b; Barro et al. 2013, 2014a; Patel et al. 2013; Stefanon et al. 2013). These galaxies have similar structural properties to the compact quiescent population, i.e., spheroidal morphologies, centrally concentrated luminosity profiles and high Sérsic indices. The similar properties and

\footnotetext{
${ }^{13}$ Hubble Fellow.
}

number densities suggest that these compact SFGs are the immediate progenitors of the similarly compact first quiescent galaxies (Barro et al. 2013). Compact SFGs are typically found in a dusty star-forming phase characterized by bright far-IR and sub-millimeter detections, and relatively normal star-formation rates (SFRs) similar to those of other SFGs of the same mass and redshift, in what is usually referred to as the star-forming main sequence (Elbaz et al. 2007; Noeske et al. 2007; Salim et al. 2007; Pannella et al. 2009; Magdis et al. 2010; Rodighiero et al. 2010; Elbaz et al. 2011; Wuyts et al. 2011a; Whitaker et al. 2012b; Pannella et al. 2015). However, they have radically different morphologies, suggesting that their compact nature is the result of strongly dissipative transformation processes, such as mergers (Hopkins et al. 2006; Naab et al. 2007; Wuyts et al. 2010; Wellons et al. 2015) or accretion-driven disk instabilities (Dekel et al. 2009; Ceverino et al. 2010; Dekel \& Burkert 2014; Zolotov et al. 2015) that funnel a large fraction of their gas reservoirs into the center, rapidly building up a dense stellar core.

Additional evidence in support of the evolutionary connection between compact SFGs and quiescent galaxies came recently when the NIR spectroscopy of a sample of compact SFGs revealed emission line widths of $\sigma=200-300 \mathrm{~km} \mathrm{~s}^{-1}$ (Barro et al. 2014b; Nelson et al. 2014), in good agreement 
with the observed stellar velocity dispersions of compact quiescent galaxies of similar stellar mass (Newman et al. 2010; Bezanson et al. 2013; van de Sande et al. 2013; Belli et al. 2014b). However, these measurements are based on different dynamical tracers, and measured on disjoint populations. Therefore, the implied evolutionary connection between them is indirect. Some caveats to this evolutionary sequence are, for example: (1) the broad emission lines may be driven by shocks and outflows rather than the gravitational potential (Newman et al. 2012b; Genzel et al. 2014), and (2) if compact SFGs do not quench immediately, their current dynamics may have little to do with their eventual transition into quiescent galaxies.

A way forward to address these issues is to study the properties of both the gas and the stars on the same galaxies. These kinematic properties can be used to test whether the gas and the stars are probing the same gravitational potential, and the simultaneous measurement of emission and absorption lines can be used to estimate the age and star-formation history (SFH) of their stellar populations. However, these kind of measurements are still observationally challenging, particularly for galaxies at $z \gtrsim 1.5$. First, absorption line measurements (e.g., the Balmer or the $\mathrm{CaHK}$ lines) require NIR spectrographs and long $(>8 \mathrm{hr}$ ) integrations on $8-10 \mathrm{~m}$ class telescopes, and probing the stronger emission lines (e.g., H $\alpha \lambda 6563 \AA$, $\left[\mathrm{N}_{\text {II }}\right] \lambda 6584 \AA,\left[\mathrm{O}_{\text {III }}\right] \lambda 5007 \AA, \mathrm{H} \beta \lambda 4861 \AA$ ) on the same galaxies often requires additional observations in other bands. Second, the spectra of galaxies are typically dominated by either emission or absorption lines, and the phases in which both sets of lines are strong are usually short and depend on the SFH of the galaxy.

Interestingly, several papers have identified a substantial population of recently quenched (i.e., young) compact quiescent galaxies at $z \gtrsim 1.5$ which are the most promising candidates to have both absorption lines from an old, underlying population and emission lines from weak, residual star formation (Newman et al. 2010; van Dokkum \& Brammer 2010; Onodera et al. 2012; Toft et al. 2012; Bezanson et al. 2013; Bedregal et al. 2013; van de Sande et al. 2013; Belli et al. 2014a, 2014b). Spectroscopic follow up of these galaxies have revealed prominent Balmer absorption lines and, occasionally, emission in [O II]. The [O II] 73726 +3729 doublet, however, is typically unresolved at the resolution of these observations, and thus it is not a reliable kinematic indicator. Additional observations of these galaxies for other emission lines in the $\mathrm{H} \alpha$ or [O III] ranges have so far been unsuccessful.

In this paper, we present $Y$ - and $H$-band spectroscopy of a massive $\left(\log \left(M / M_{\odot}\right)=10.75\right)$, compact SFG at $z=1.674$ obtained with the MOSFIRE multi-object infrared spectrograph (McLean et al. 2010, 2012) on the Keck I telescope. The spectra of GDN-8231 show emission and absorption lines which suggest that the galaxy is quenching rapidly. We model these lines to estimate the kinematics of the gas and the stars. In addition, we combine the spectra with optical medium-band photometry from the SHARDS survey (Pérez-González et al. 2013) and Hubble Space Telescope (HST) Wide Field Camera 3 (WFC3) grism spectroscopy in G102 (GO13420; PI = Barro) and G141 (GO11600; $\mathrm{PI}=$ Weiner) to obtain detailed spectral energy distributions (SEDs) for the quenching galaxy and three older quiescent galaxies, observed as a part of the same MOSFIRE survey.
From the modeling of their SEDs, we estimate their stellar ages and SFHs, and analyze the implications for the evolutionary path from compact SFG to compact quiescent galaxy.

Throughout the paper, we adopt a flat cosmology with $\Omega_{M}=0.3, \Omega_{\Lambda}=0.7$ and $H_{0}=70 \mathrm{~km} \mathrm{~s}^{-1} \mathrm{Mpc}^{-1}$ and we quote magnitudes in the $\mathrm{AB}$ system.

\section{DATA}

\subsection{Target Selection}

We select spectroscopic targets from the CANDELS (Grogin et al. 2011; Koekemoer et al. 2011) WFC3/F160W ( $H$-band) multi-wavelength catalogs in GOODS-N (G. Barro et al. 2016, in preparation). This catalog includes deep, UV-to-NIR ground-based observations in several medium and broad bands, HST photometry in nine different bands, and Spitzer/ Herschel observations in the mid-to-far-IR. The source extraction and merged multi-wavelength SEDs are measured following the procedures described in Galametz et al. (2013) and Guo et al. (2013). In addition, GOODS-N includes complementary HST/WFC3 observations in both the G102 and G141 grisms, allowing for continuous wavelength coverage from $0.9<\lambda<1.7 \mu \mathrm{m}$ with a resolution better than $R=130$ (e.g., Brammer et al. 2012). The grism data are reduced using the threedhst ${ }^{14}$ pipeline. The pipeline handles the combination and reduction of the dithered exposures, and the extraction of the individual spectra. These are backgroundsubtracted and corrected for contamination from the overlapping spectra of nearby sources (Brammer et al. 2012; Momcheva et al. 2015). The grism data are joined at shorter wavelengths by the 25 optical medium bands of the SHARDS survey ( $R \sim 50$; Pérez-González et al. 2013). Together, these data sets provide remarkable spectral resolution on a galaxyby-galaxy basis that is uniquely suited for SED-fitting analysis (see Section 3). The stellar masses, rest-frame colors, and photometric redshifts used for target selection are derived via SED fitting using EAzY (Brammer et al. 2008) and FAST (Kriek et al. 2009), assuming Bruzual \& Charlot (2003) stellar population synthesis models, a Chabrier (2003) initial mass function (IMF), and the Calzetti et al. (2000) dust extinction law. The SFRs are computed by adding the unobscured and obscured star formation, traced by the UV and IR emission, following the method described in Wuyts et al. (2011a; see also Barro et al. 2013, 2014a)

We select galaxies in a transition stage between the starforming main sequence and the quiescent, red sequence using $-0.75<\log \left(\mathrm{sSFR} / \mathrm{Gyr}^{-1}\right)<-0.25$. This corresponds to galaxies approximately $\sim 2 \sigma$ below the main sequence at $z \sim 1.75$ (Whitaker et al. 2012b). For galaxies with $\log \left(M / M_{\odot}\right)>10$, this threshold roughly corresponds to a SFR $\gtrsim 10 M_{\odot} \mathrm{yr}^{-1}$, which is the $5 \sigma$ detection level with MOSFIRE in $\sim 1 \mathrm{hr}$ exposures (e.g., Trump et al. 2013; Kriek et al. 2015). We also impose the compactness criterion of Barro et al. (2013), $M / r_{\mathrm{e}}^{1.5}=10.3 M_{\odot} \mathrm{kpc}^{-1.5}$, to select galaxies with similar structural and morphological properties as the quiescent population at that redshift. Figure 1 illustrates that the sSFR criterion is consistent with the $U-V$ versus $V-J$ (hereafter $U V J)$ rest-frame color criterion that has been shown to be very successful in identifying quiescent galaxies, breaking the age/ dust degeneracy (Wuyts et al. 2007; Williams et al. 2010;

\footnotetext{
${ }^{14}$ http://code.google.com/p/threedhst
} 

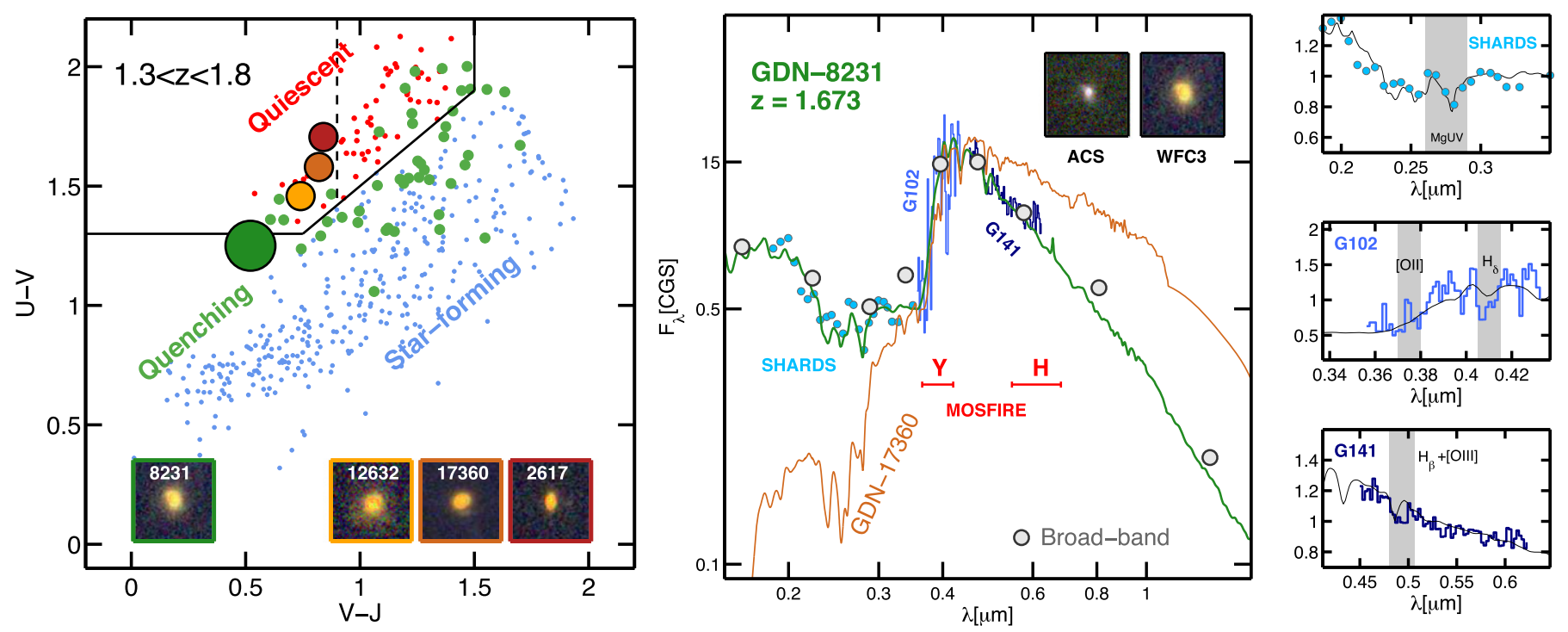

Figure 1. Left panel: $U V J$ diagram for galaxies at $1.3<z<1.8$ more massive than $\log \left(M / M_{\odot}\right)>10$ in the GOODS-N field. The colors highlight different populations of star-forming galaxies (blue; $\left.\log \left(\mathrm{sSFR} / \mathrm{Gyr}^{-1}\right)>-0.25\right)$, quiescent galaxies (red; $\log \left(\mathrm{sSFR} / \mathrm{Gyr}^{-1}\right.$ ) $<-0.75$ ), and quenching transition galaxies (green; $-0.25<\log \left(\mathrm{sSFR} / \mathrm{Gyr}^{-1}\right)<-0.75$ ) identified according to their UV+IR SFRs. The large markers show the quenching, compact SFG (GDN-8231; dark green), and the three quenched galaxies (GDN-2617, GDN-17360, and GDN-12632; orange to red) observed with MOSFIRE. The HST color images ( $z J H)$ of the four galaxies are shown at the bottom. The location of GDN-8231 in the $U V J$ is consistent with the observed spectral and photometric properties, indicating that it is a weakly star-forming galaxy transitioning to a quiescent phase. The quiescent galaxies fall within the selection region for recently quenched galaxies (left of the dashed line; Whitaker et al. 2012a). Right panel: color images (ACS and WFC3), and composite SED of GDN-8231. The gray circles show the (low-resolution) broad-band photometry, the cyan markers show the SHARDS medium-band data $(R \sim 50$; Pérez-González et al. 2013), and the light and dark blue lines show the HST/WFC3 G102 and G141 grism spectra. The spectral regions probed by $Y$ - and $H$-band MOSFIRE spectra are indicated in red. The green and orange lines show the best-fit stellar population templates from Pacifici et al. (2012) at a resolution of $R=50$ for GDN-8231 and the quiescent galaxy GDN-17360. The latter illustrates that despite their relatively similar $U V J$ colors, GDN-8231 has a clearly different SED typical of a post-starburst galaxy. The three sub-panels on the right show the zoom-in around the SHARDS, G102, and G141 data highlighting spectral features in $\mathrm{Mg}_{\mathrm{UV}}$, [O II], $\mathrm{H} \delta$, and $\mathrm{H} \beta$.

Whitaker et al. 2011). The spread of the transition sample along the wedge of the UVJ quiescent region is primarily driven by differences in the dust reddening and in the stellar population ages, which suggests a wide diversity of extinction levels and SFHs among quenching galaxies (e.g., Wild et al. 2014).

We select the most promising candidates for spectroscopic follow up by prioritizing bright galaxies with low dust reddening to maximize the signal-to-noise ratio $(\mathrm{S} / \mathrm{N})$. This additional restriction preferentially selects galaxies with bluer $U V J$ colors, near the so-called post-starburst region of the $U V J$ diagram (left of the dashed line in Figure 1; Whitaker et al. 2011; Bezanson et al. 2013). This region encompasses colors that are typical of recently quenched galaxies, with low extinction levels and young stellar ages of $\lesssim 1 \mathrm{Gyr}$. Galaxies in this region also have, on average, lower stellar masses $\left(\log \left(M / M_{\odot}\right) \lesssim 10.8\right)$ than older quiescent galaxies with redder colors (Newman et al. 2015; Barro et al. 2014a). Thus, we caution that this sample is not entirely representative of all quenching (green circles) or quenched galaxies (red circles) at this redshift.

The redshift is restricted to the range $1.3<z<1.8$ that places the $\mathrm{H} \alpha$ emission line in the $H$ band and several other Balmer lines around the $4000 \AA$ break in the $Y$ band. The observed mask contains one candidate that is a transitioning compact SFG (GDN-8231; green circle) and three recently quenched galaxies (GDN-12632, GDN-17360, and GDN-2617; orange to red circles). GDN-8231 is relatively massive $\left(\log \left(M / M_{\odot}\right)=(10.7)\right.$ and presents low levels of star formation (SFR $=10 M_{\odot} \mathrm{yr}^{-1}$ ) evidenced by a weak detection in MIPS $24 \mu \mathrm{m}(f(24)=40 \mu \mathrm{Jy})$. The low sSFR and mild extinction $\left(A_{\mathrm{V}}=0.3 \mathrm{mag}\right)$ are consistent with its location in the $U V J$ diagram, and suggests that it is on the brink of becoming a young quiescent galaxy, similar to the three quiescent targets in our sample. The photometric and spectroscopic galaxy properties of the four galaxies are summarized in Table 1. In passing, we note that three of the four galaxies in our sample have very close spectroscopic redshifts, which suggest that they may be in a dense environment. Further NIR spectroscopic follow up in areas of GOODS-N with likely overdensities will provide additional evidence to verify this possibility, e.g., from surveys like MOSDEF (Kriek et al. 2015) or ZFIRE (Tran et al. 2015).

\subsection{Spectroscopic Data}

Data were collected on the nights of 2014 April 17 and May 11 using the MOSFIRE instrument (McLean et al. 2010, 2012) on the Keck I telescope. The sky conditions were clear and the median seeing ranged from 0 ". 5 to 0 ". 7 seeing. We observed one mask configuration in both the $Y(0.97<\lambda<1.12 \mu \mathrm{m})$ and $H$ bands $(1.46<\lambda<1.81 \mu \mathrm{m})$, with individual exposure times of $180 \mathrm{~s}$ and $120 \mathrm{~s}$, for a total $5.5 \mathrm{hr}$ and $2 \mathrm{hr}$, respectively. We used two-point dithers separated by 1.15 and slit widths of 0 ". 7 . The instrumental resolution of MOSFIRE with 0 ".7 slit widths is approximately $R=3200$ ( $\sim 5 \AA$ per resolution element). The two-dimensional (2D) spectra were reduced, sky subtracted, wavelength-calibrated, and onedimensionally extracted using the public MOSFIRE data reduction pipeline. ${ }^{15}$

\footnotetext{
${ }^{15}$ http://www2.keck.hawaii.edu/inst/mosfire/drp.html
} 
Table 1

Stellar and Spectroscopic Properties of the MOSFIRE Sample

\begin{tabular}{|c|c|c|c|c|c|c|c|c|c|c|c|c|c|c|}
\hline $\begin{array}{l}\text { ID } \\
(1) \\
\end{array}$ & $\begin{array}{l}\text { R.A. } \\
\text { (2) }\end{array}$ & $\begin{array}{c}\text { Decl. } \\
(3)\end{array}$ & $\begin{array}{c}z_{\text {spec }} \\
(4) \\
\end{array}$ & $\begin{array}{c}\text { F160W } \\
(5)\end{array}$ & $\begin{array}{c}\log M_{\star} \\
\text { (6) }\end{array}$ & $\begin{array}{c}r_{e} \\
(7) \\
\end{array}$ & $\begin{array}{c}n \\
(8) \\
\end{array}$ & $\begin{array}{l}\sigma_{\star} \\
(9) \\
\end{array}$ & $\begin{array}{l}\sigma_{\mathrm{gas}} \\
(10) \\
\end{array}$ & $\begin{array}{c}\mathrm{EW}(\mathrm{H} \alpha) \\
(11)\end{array}$ & $\begin{array}{l}\mathrm{H} \delta_{\mathrm{A}} \\
(12) \\
\end{array}$ & $\begin{array}{c}\mathrm{EW}(\mathrm{H} \delta) \\
(13)\end{array}$ & $\begin{array}{c}\text { D4000 } \\
(14)\end{array}$ & $\begin{array}{l}\text { Age } \\
(15) \\
\end{array}$ \\
\hline 8231 & 189.0656 & 62.1987 & 1.674 & 21.40 & 10.75 & 2.48 & 3.95 & $215 \pm 35$ & $127 \pm 32$ & $5.9_{-0.7}^{+1.1}$ & $3.6_{-0.4}^{+0.5}$ & $2.5 \pm 2.0$ & $1.45 \pm 0.02$ & $0.75 \pm 0.12$ \\
\hline $12632^{\mathrm{a}}$ & 188.9625 & 62.2286 & 1.598 & 21.25 & 11.02 & 1.32 & 8.00 & $177 \pm 34$ & $\cdots$ & $-0.4_{-0.8}^{+0.6}$ & $4.3_{-1.0}^{+1.1}$ & $\cdots$ & $1.59 \pm 0.03$ & $1.07 \pm 0.20$ \\
\hline 17360 & 189.1153 & 62.2594 & 1.674 & 21.75 & 10.78 & 1.12 & 3.31 & $146 \pm 29$ & $\cdots$ & $-0.7_{-1.1}^{+0.5}$ & $3.0_{-2.0}^{+1.2}$ & $5.8 \pm 4.5$ & $1.62 \pm 0.03$ & $1.14 \pm 0.15$ \\
\hline 2617 & 189.1003 & 62.1532 & 1.675 & 22.07 & 10.61 & 0.87 & 2.04 & $295 \pm 110$ & $\ldots$ & $0.0_{-1.5}^{+0.5}$ & $2.6_{-1.4}^{+0.7}$ & $3.6 \pm 3.7$ & $1.65 \pm 0.03$ & $1.31 \pm 0.27$ \\
\hline
\end{tabular}

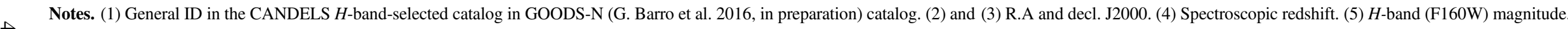

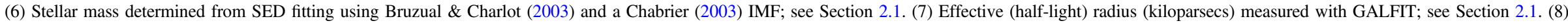

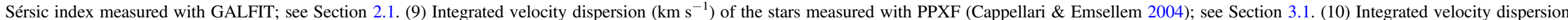

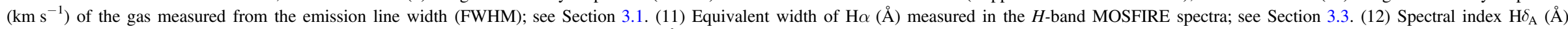

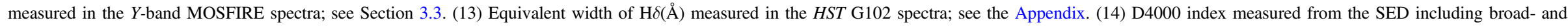
medium-band photometry as well as HST grism spectroscopy; see Section 3.3. (15) Luminosity-weighted stellar age (gigayears) estimated from SED fitting using the models of Pacifici et al. (2012).

${ }^{a}$ Observed in Newman et al. (2010), Belli et al. (2014a), and LRIS optical spectroscopy with $\sigma=174 \mathrm{~km} \mathrm{~s}^{-1}$. 

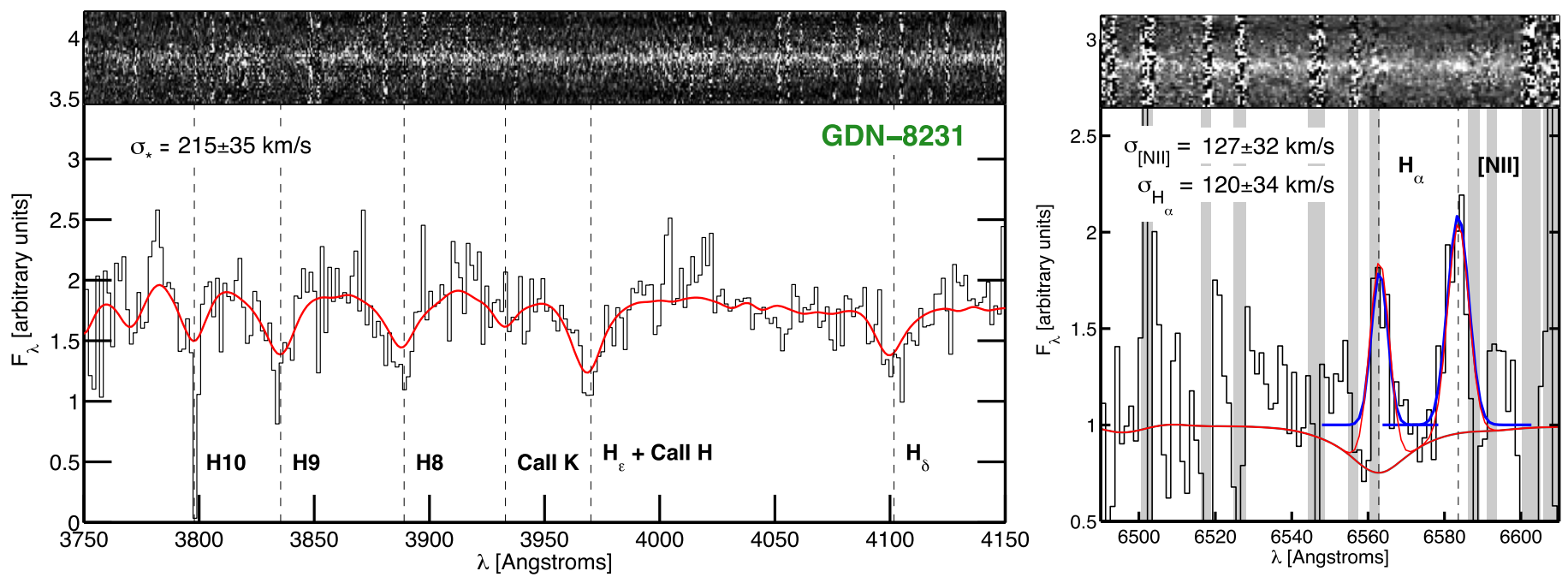

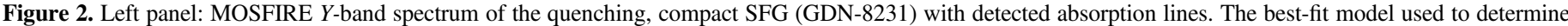

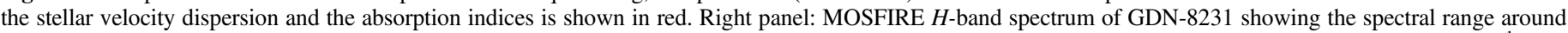

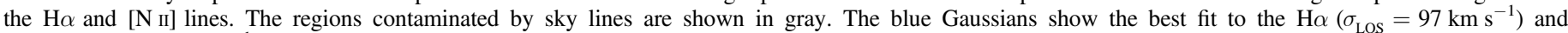

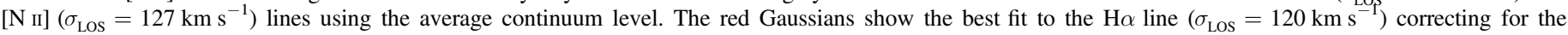
$\mathrm{H} \alpha$ stellar absorption as determined from the fit to the Y-band spectrum.

\section{ANALYSIS}

\subsection{Kinematic Measurements and Line Ratios}

The four galaxies are NIR-bright $(H<22 \mathrm{mag})$ and present clear continuum detections $(\mathrm{S} / \mathrm{N}>5)$ in both the $Y$ - and $H$ band spectra. GDN-8231 exhibits multiple Balmer absorption lines in the $Y$-band spectrum, from $\mathrm{H} \delta$ to $\mathrm{H} 10$, and it is the only galaxy showing $\mathrm{H} \alpha$ and $[\mathrm{N}$ II] emission lines in the $H$-band spectrum (Figure 2). The other three quiescent galaxies also have clear Balmer absorption lines in the $Y$ band but present no emission lines in the $H$ band (Figure 8 of the Appendix).

We measure the line-of-sight (LOS) stellar velocity dispersion using the penalized pixel-fitting software pPXF (Cappellari \& Emsellem 2004). This software fits the galaxy spectrum with a model created as a linear combination of the stellar templates that best reproduce the galaxy spectrum allowing different weights for each templates. The stellar templates used are the stars from the MILES stellar library (Sánchez-Blázquez et al. 2006b; Cenarro et al. 2007). Before fitting the galaxy spectrum, we mask the regions contaminated by telluric atmospheric bands and pixels with strong residuals from the sky lines, and convolve it with a Gaussian function whose width is the quadratic difference between the resolution of the MILES stellar library (FWHM $=2.5 \AA$; Falcón-Barroso et al. 2011) and the instrumental resolution of our observations measured in sky lines $(\mathrm{FWHM}=2.7 \AA)$. We estimate the uncertainty in the stellar velocity dispersion by running 1000 Monte Carlo simulations. In each simulation, the flux of the spectrum is perturbed within a Gaussian function whose width is the uncertainty in the flux obtained in the reduction process. The stellar velocity dispersion is measured in each simulation and the uncertainty is the standard deviation of the Gaussian distribution of all 1000 velocity dispersion estimates. Based on this method, we obtain a stellar velocity dispersion for GDN8231 of $\sigma^{\star}=215 \pm 35 \mathrm{~km} \mathrm{~s}^{-1}$ (see Table 1 for the quiescent galaxies).

We verify the robustness of our velocity dispersion measurements by doing the following tests: (1) we check the influence of the wavelength range used in the fitting procedure
(3700 $<\lambda<4200)$ by making it smaller. We restrict the wavelength coverage to a range with only two absorption lines, typically $300 \AA$, and find offsets in the velocity dispersion that are $<10 \%$ both in the positive and negative directions, which indicates that there are no systematic biases. These values are within the $1 \sigma$ uncertainties, which are typically $\sim 20 \%$ for the galaxies studied here. We quote our velocity dispersions measured in the full range of $500 \AA$; (2) we check the influence of the stellar templates by comparing our results based on the MILES stellar library with those based on the ELODIE and INDOUS stellar libraries (Valdes et al. 2004; Prugniel et al. 2007, respectively). The velocity dispersions obtained agree well within the uncertainties. These tests are consistent with the previous analysis performed by van de Sande et al. (2013) and Belli et al. (2014a, 2014b).

We measure the LOS gas velocity dispersion, $\sigma_{\text {LOS }}^{\text {gas }}$, of GDN8231 by fitting a Gaussian profile to the emission lines, measuring its FWHM, and subtracting the instrumental broadening in quadrature from the FWHM. The velocity dispersion is then the corrected FWHM divided by 2.355 . We fit the $\mathrm{H} \alpha$ and [ $\mathrm{N}_{\text {II }}$ lines independently. As shown in Figure 2, [N $\mathrm{N}_{\mathrm{II}}$ is detected at higher $\mathrm{S} / \mathrm{N}$ because the $\mathrm{H} \alpha$ line is partially contaminated by a skyline, and it appears to be self-absorbed (i.e., the continuum emission is affected by Balmer absorption). As a result, if we fit the lines using the same continuum level, the velocity dispersion inferred from $\mathrm{H} \alpha$ is smaller than that from [N II], $\sigma_{\text {LOS }}^{\text {gas }}=90 \pm 18 \mathrm{~km} \mathrm{~s}^{-1}$ and $127 \pm 32 \mathrm{~km} \mathrm{~s}^{-1}$, respectively. Although it is possible for Balmer and forbidden lines to have different widths if they originate in different regions, the most likely explanation for such a large difference is the self-absorption in $\mathrm{H} \alpha$. To account for that effect, we use the best-fit stellar template to the absorption spectra to establish the continuum level for the $\mathrm{H} \alpha$ emission, and we recompute the fit. With this method, the inferred velocity dispersion is $\sigma_{\mathrm{LOS}}^{\text {gas }}=120 \pm 34 \mathrm{~km} \mathrm{~s}^{-1}$, consistent with the $[\mathrm{N}$ II $]$ result. Although the two measurements agree after accounting for Balmer absorption, we adopt the higher $\mathrm{S} / \mathrm{N}$ $[\mathrm{N}$ II] measurement as the more reliable tracer of gas velocity dispersion. 
The spectra are not flux-calibrated. However, we can use the equivalent width of $\mathrm{H} \alpha$ corrected for stellar absorption (EW $\left.(\mathrm{H} \alpha)_{\mathrm{corr}}=8.3 \pm 0.7\right)$ and the continuum flux, inferred from the SED modeling, to calculate the $\mathrm{H} \alpha$ line flux and SFR. Using the empirical relation from Kennicutt (1998) and the attenuation inferred from SED fitting $\left(A_{\mathrm{V}}=0.3\right)$, we obtain values of SFR $=3-6 M_{\odot} \mathrm{yr}^{-1}$, depending on the extra nebular extinction with respect to the continuum $\left(A_{\mathrm{H} \alpha}=2.44-1.86\right.$ $A_{\text {cont }}$; e.g., Calzetti et al. 2000; Price et al. 2014). These values are consistent with the estimate from MIPS $24 \mu \mathrm{m}$ data. The $\mathrm{H} \alpha$ line flux corrected for stellar absorption also allows us to estimate the intrinsic value of the line ratio $[\mathrm{N}$ II] $/ \mathrm{H} \alpha=1.2$. Even without measuring the $\left[\mathrm{O}_{\mathrm{III}}\right] / \mathrm{H} \beta$ line ratio to fully constrain the ionization diagnostic diagram (i.e., BPT; Baldwin et al. 1981), an [N II] $/ \mathrm{H} \alpha$ ratio of the order of unity already suggests that the nebular emission is at least partially powered by an active galactic nucleus (AGN). The galaxy, however, is not detected in the 2Ms X-ray data (Alexander et al. 2003), which implies that the AGN is relatively weak, perhaps shutting down along with the star formation in the galaxy. High values of the $[\mathrm{N}$ II $] / \mathrm{H} \alpha$ ratio in galaxies with suppressed or fully quenched star formation are typical of LINER galaxies (e.g., Yan et al. 2006), and has also been recently observed on a massive quiescent galaxy at $z \sim 2$ (Newman et al. 2015). This result, together with the large fraction of AGNs $(\sim 40 \%)$ found in compact SFGs at $z=2-3$ (Barro et al. 2014a), suggest that the black hole growth at high redshift closely follows the SFH (i.e., it starts and shuts down with the star formation), as opposed to the observed trend in the local universe, where there seems be to a delay between the starburst and the peak of the AGN phase (Wild et al. 2010; Hernán-Caballero et al. 2014; Yesuf et al. 2014). In that case, AGNs may play a more active role in the quenching of star formation at $z \sim 2$.

\subsection{Luminosity Profile and Morphology}

The MOSFIRE spectra of GDN-8231 are spatially unresolved and thus do not provide additional insights on the kinematic profile of the gas and the stars, or the spatial distribution of star formation. The high-resolution HST imaging, however, can be used to determine the overall structural properties and the radial distribution of star formation in the galaxy. Figure 3 shows the surface brightness and color profiles of GDN-8231 computed from the fitting of the HSTbased SEDs measured at each radius. The profile shows a positive color gradient of $d(\mathrm{NUV}-V) / d r=6 \cdot 10^{-3} \mathrm{mag} /$ $\mathrm{kpc}$ that is indicated by a bluer center with a $20 \%$ smaller $r_{e}$ in the rest-frame NUV with respect to the $V$ band $\left(r_{e, \mathrm{NUV}} / r_{\mathrm{e}, \mathrm{V}}=0.8\right)$. Assuming that the NUV is a good tracer of the SFR, these measurements suggest that the SFR profile is more centrally concentrated than the stellar profile. A possible interpretation for this result is that we are witnessing the quenching of a central starburst right after it has formed a dense stellar core $\left(\Sigma_{1 \mathrm{kpc}}=9.7 M_{\odot} \mathrm{kpc}^{-2}\right)$, which seems to be a prerequisite for quiescent galaxies (Cheung et al. 2012; Fang et al. 2013; van Dokkum et al. 2014). Such interpretation is an excellent match for the predictions of simulations that describe the formation of compact galaxies as the result of strongly dissipative gas-rich events, such as mergers and/or disk instabilities (e.g., Dekel \& Burkert 2014; Wellons et al. 2015; Zolotov et al. 2015). These processes are characterized by a wet inflow (i.e., gas-inflow rate $>$ SFR) that builds up the central gas density, thereby enhancing the SFR at the center

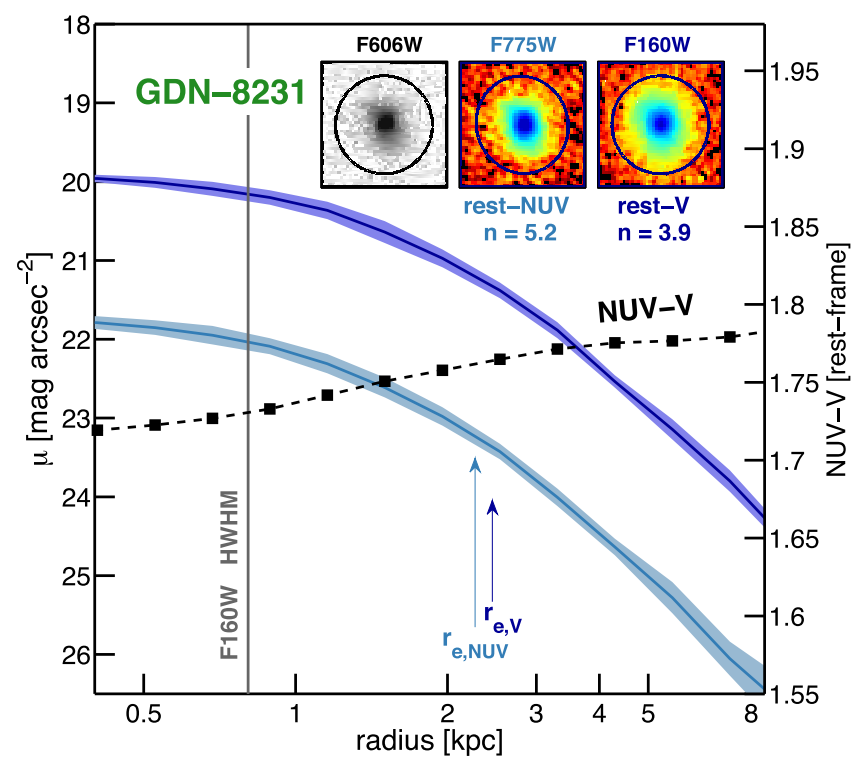

Figure 3. Rest-frame surface brightness profile and color profile of GDN-8231. The rest-frame profiles are computed by interpolating at each radius the best-fit SED derived from the observed surface brightness profile in nine HST bands measured with IRAF/ellipse (see Liu et al. 2013 and F. Liu et al. 2016, in preparation, for more details). At $z=1.67$, the rest-frame NUV and V bands roughly correspond with the observed $i$ and $H$ bands, respectively. The arrows indicate the effective radius in those bands from the best-fit Sérsic profiles obtained using GALFIT. The gray line indicates the point-spread function (PSF) half width at half-maximum (HWHM) in the $H$ band. The insets show the images of GDN-8231 in the $i$ (PSF-matched to the $H$ band) and $H$ bands. The black circle has radius of $1^{\prime \prime}(\sim 8.4 \mathrm{kpc})$. GDN-8231 has an positive color gradient and relatively low sSFR, which are consistent with the expectations for an early phase of inside-out quenching after a central starburst.

and growing a dense stellar core. The weakening of such inflow marks the onset of inside-out quenching due to gas depletion. In that onset phase, the SFR is still high and thus the color gradient is still positive, as observed in GDN-8231. However, as inside-out quenching progresses, the SFR profile flattens, and the color gradient turns negative, as observed in compact quiescent galaxies (Guo et al. 2012; Szomoru et al. 2012; Wuyts et al. 2012).

GDN-8231 has high-Sérsic values consistent with other compact star-forming and quiescent galaxies (Barro et al. 2013). Its visual appearance in the $H$ band is smooth and spheroidal. However, the ACS image (F606W gray-scale in Figure 3), that probes the rest-frame UV, shows a slight lowsurface brightness asymmetry $\left(\mu<26 \mathrm{mag} \operatorname{arcsec}{ }^{2}\right)$ in the north-east area of the profile of the galaxy at $r 6 \mathrm{kpc}$. Such irregularities could be indicative of a late-stage compaction/ merger event.

In spite of the high Sérsic value of the stellar profile of the galaxy, it is possible that the gas and the star formation are distributed in a disk component. We discuss this possibility in more detail in Section 4.2 where we estimate the kinematics of the gas from the analysis of the emission line spectrum. Such analysis requires an estimate of the projection effects along the line of sight, which are usually determined by means of the observed axial ratio of the stellar component, $q=b / a$. Assuming that disks are oblate spheroids, then the inclination angle can be corrected for the effects of the intrinsic thickness of the disk: $\cos ^{2} i=\left(q^{2}-q_{0}^{2}\right) /\left(1-q_{0}^{2}\right)$. Here $q_{0}$ is the intrinsic axial ratio of the disk when observed edge-on. It is customary to assume a constant value of $q_{0} \sim 0.2$ (see, e.g., 
Courteau et al. 2007; Miller et al. 2011), although some authors argue that this value could decrease toward $q_{0} \sim 0.1$ in latetype spirals (Haynes \& Giovanelli 1984; Dale et al. 1997). Adopting these assumptions, we infer a viewing angle inclination of $i=42^{\circ}$ from an axis-ratio value of $b / a=0.74$.

\subsection{Spectral Indices and SED Fitting}

One of the main obstacles to estimating stellar population properties from the analysis of SEDs is that at the typical resolution of broad-band surveys (FWHM $\sim 0.2 \mu \mathrm{m} /(1+z)$; $R \sim 6)$ the most relevant continuum and emission/absorption line features are usually diluted, which results in large uncertainties in inferred properties (Muzzin et al. 2009; Conroy \& Gunn 2010). One way to circumvent this problem is by using higher spectral resolution data to obtain more accurate measurements of line strengths and spectral indices, which are key indicators of stellar age and present/past star-formation activity (e.g., Kelson et al. 2001; Kauffmann et al. 2003).

From the MOSFIRE $Y$-band spectra we measure the $\mathrm{H} \delta_{\mathrm{A}}$ Lick index (Worthey \& Ottaviani 1997). The uncertainty in this value is estimated by running 1000 Monte Carlo simulations. In each simulation, the flux of the target galaxy is randomly perturbed within a Gaussian function whose width is the difference between the galaxy spectrum and the best-fit composite stellar template used to obtain the stellar kinematics. In addition, the perturbed galaxy spectrum is convolved with a Gaussian function whose width is randomly chosen within the $1 \sigma$ uncertainty of the velocity dispersion of the galaxy, and it is shifted in wavelength by a randomly chosen value within the $1 \sigma$ uncertainty of the galaxy redshift. The uncertainty in the $\mathrm{H} \delta_{\mathrm{A}}$ index is the standard deviation of the Gaussian distribution of the 1000 Monte Carlo simulations. This index has been traditionally used as an age and SFH indicator (Trager et al. 2000; Sánchez-Blázquez et al. 2006a). From the $H$-band spectra we measure the $\mathrm{H} \alpha$ equivalent width, $\mathrm{EW}(\mathrm{H} \alpha)$, which is sensitive to the instantaneous (last few megayears) SFR. Only GDN-8231 presents $\mathrm{H} \alpha$ emission; the other three quiescent galaxies have strong continuum detections that place reliable upper limits on $\mathrm{EW}(\mathrm{H} \alpha)$. In addition to the MOSFIRE spectra, we use the SHARDS medium-band data and the HST grism data to probe for additional absorption features and continuum breaks. The right panel of Figure 1 illustrates the high spectral resolution $(\sim 10-100 \times$ better than broad-band filters) of the merged medium-band/grism SED, revealing Mg II absorption at $2800 \AA$ in SHARDS and Balmer absorption lines and the $4000 \AA$ break in G102 and G141. We quantify the later measuring the D4000 index on the MOSFIRE data (e.g., Balogh et al. 1999; Kauffmann et al. 2003). The G102 spectrum of GDN-8231 also shows a weak [O II] emission line (EW $15 \pm 5 \AA$ ). However, there is no clear sign of [O III] emission in G141. The later is expected to also have low $\mathrm{EW} \lesssim 10 \AA$, and thus can be partially hidden by the $\mathrm{H} \beta$ absorption, as hinted in the stacked spectra of recently quenched galaxies in Whitaker et al. (2013).

The spectral indices are usually analyzed by comparing measurements to a grid of values derived from stellar population synthesis models. Here we follow a slightly different approach to combine information from both the indices and the overall UV-to-NIR SED by using of the SEDfitting code of Pacifici et al. (2012, hereafter P12). The code performs a simultaneous fitting of the low- and high-resolution data and includes priors on the EW of emission and absorption lines to obtain better constraints on the SFR and SFH of the galaxy (see also Pacifici et al. 2015 and Barro et al. 2014a for more details). The galaxy templates are computed from nonparametric SFHs adapted from semi-analytic models (SAMs) and include both the stellar continuum and the nebular emission. The SAM SFHs provide a richer parameter space featuring short-timescale variations of the SFR (burst and truncations) that are missing in exponentially declining (exp $[-t / \tau])$ or delayed $(t \times \exp [-t / \tau])$ models (see also Section 4.1).

\section{RESULTS}

\subsection{Stellar Ages and SFHs}

The stellar age and SFH are not independent properties and thus the chosen parametrization of the latter (single burst, N-bursts, $\tau$ models, SAMs, etc.) often leads to strong degeneracies in the age and the characteristic timescale(s) of star formation (e.g., age- $\tau$; Conroy 2013). These degeneracies, however, can be reduced using higher spectral resolution data sets to probe features that are sensitive to different star formation and quenching timescales (Kriek et al. 2011; Pérez-González et al. 2013; Belli et al. 2015).

Figure 4 illustrates the implications of assuming different SFHs to estimate the age of the galaxies. The blue shaded region shows the evolution of the sSFR and luminosityweighted age versus time for galaxies in the star-forming main sequence, as described by the SAM SFHs in the model library of Pacifici et al. (2012). Those galaxies are thought to be growing in a relatively smooth, secular mode (Elbaz et al. 2007; Rodighiero et al. 2010) in which gas inflow and SFR have reached a steady-state phase (e.g., Dekel et al. 2013). The main sequence pictured by SAM SFHs closely follows the observational results (e.g., Whitaker et al. 2014), which are also in good agreement with the predictions of other empirical models (e.g., Peng et al. 2010; Behroozi et al. 2013). In the main sequence paradigm, quenching can be interpreted as the departure from a stable growth phase, which can be either fast (e.g., SFR truncation; solid line) or slow (shallower slope; dashed line). After fast quenching, age increases linearly with time (i.e., passive evolution), while for slower quenching the slope is shallower, and the age increases slowly due to slowly declining star formation (right panel of Figure 4). In $\tau$ models, the timescale controls the quenching time, and it can be tuned to describe fast or slow quenching (gray line in Figure 4). However, as one parameter characterizes the whole SFH, the prediction is not realistic for galaxies that are described by two or more distinct phases, such as a secular growth followed by fast quenching.

Panels (a) and (b) of Figure 5 show index-index diagrams sensitive to the SFH and quenching time. The colored circles indicate the values measured in the MOSFIRE spectra of the four galaxies, and the lines show the evolutionary tracks of their best-fit SAM SFHs. The D4000 index is a good tracer of the average age of the stellar populations, while the $\operatorname{EW}(\mathrm{H} \alpha)$ and $\mathrm{H} \delta_{\mathrm{A}}$ are more sensitive to recent star formation. For slow quenching, the $\mathrm{EW}(\mathrm{H} \alpha)$ in emission decreases slowly while the Balmer absorption is relatively small and constant with time, $\mathrm{H} \delta_{\mathrm{A}} \sim 1.8$. In contrast, fast quenching causes a rapid decline in the $\mathrm{H} \alpha$ emission followed by an absorption plateau with $\operatorname{EW}(\mathrm{H} \alpha) \sim-2 \AA$, and a strong $\mathrm{H} \delta_{\mathrm{A}}$ absorption. This relatively short-lived phase ( $\sim$ Gyr), characterized by the lack 

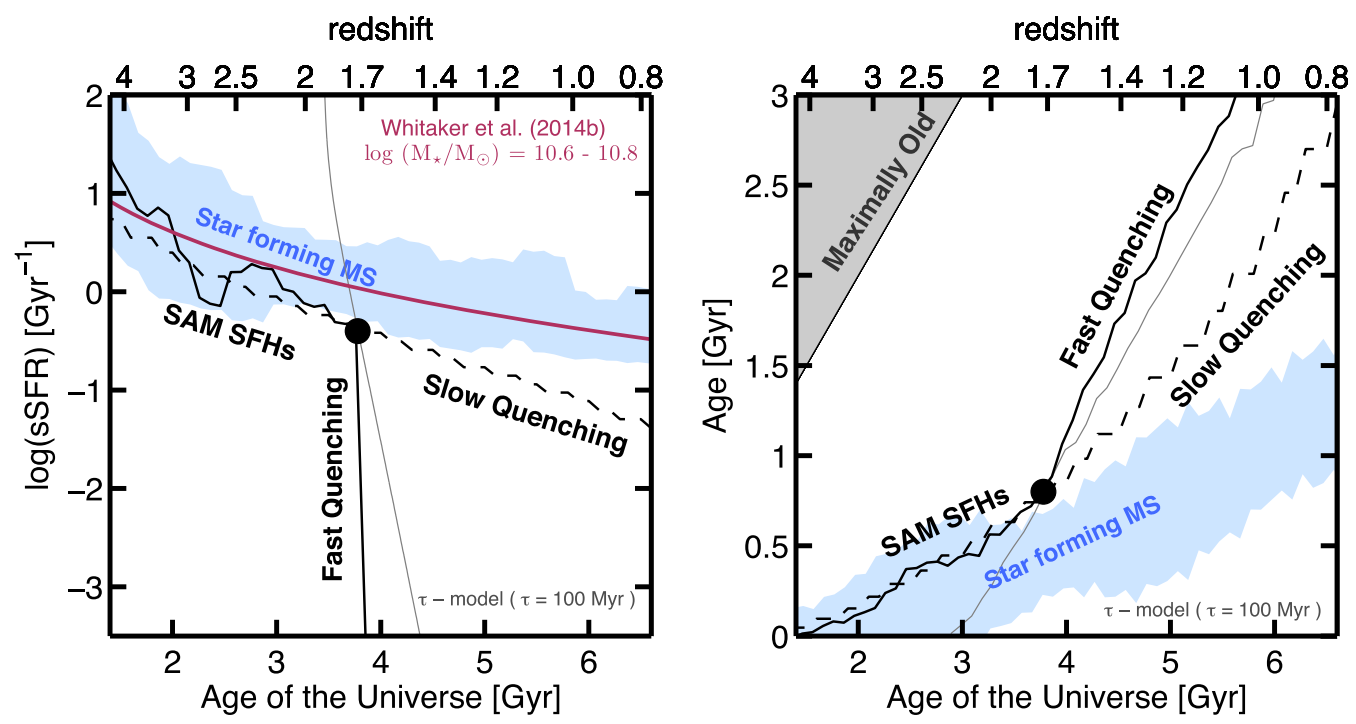

Figure 4. Evolutionary tracks in sSFR (left) and luminosity-weighted age (right) vs. age of the universe for different SFHs. The blue shaded region depicts the starforming main sequence determined from the average SFH of SFGs drawn from the model library of Pacifici et al. (2012). This region agrees well with the observational results of Whitaker et al. (2014) for SFGs of intermediate mass. The black lines illustrate the evolution of two galaxies that have a secular growth from $z \sim 4$ to $z=1.7$, followed by either fast (solid) or slow (dashed) quenching of star formation. In a fast-quenching galaxy, the luminosity-weighed age grows linearly with time (passive evolution). However, in a slow-quenching (or main sequence) galaxy, the luminosity-weighed age increases more slowly (i.e., the slope is $<1$ ). A $\tau$ model can describe either a fast or slow quenching. However, a short $\tau$ (gray line) would provide unrealistic results for galaxies described by a two-phase SFH (e.g., a main sequence + fast quenching).

of $\mathrm{H} \alpha$ emission and strong absorption in other Balmer lines, typical of A-stars, is known as a post-starburst phase (pSB; e.g., Wild et al. 2010). This phase is clearly identified in the $\mathrm{H} \delta_{\mathrm{A}}$ versus D4000 diagram as the "hump" above the dashed black line which indicates a slowly declining SFH.

In summary, SFGs are typically located within the blue regions of the SFR-MS in panels (a) and (b). Quenching(-ed) galaxies are located either along the dashed black line, if they experienced a slow decline of the SFR, or below/above the dashed line in panels (a) and (b) if they experienced a fast quenching.

The spectral properties of GDN-8231 agree well with the sSFR selection criteria, indicating that the galaxy is on a fastquenching path. Besides the $\mathrm{H} \alpha$ emission, which indicates the presence of weak, ongoing star formation, the SED fit, the D4000 index, and the $\mathrm{H} \delta_{\mathrm{A}}$ values suggest that the emission is rapidly declining. In fact, given the short quenching time, the chances of finding a galaxy in this phase are small, which makes GDN-8231 quite unique. The three quiescent galaxies in our sample are further along in their evolution, as indicated by their stronger D4000 1.6. However, their indices and best-fit SFHs are also consistent with fast quenching. The similar SFHs are another indication that GDN-8231 is evolutionarily linked to the quenched galaxies. For a definition of quenching time, $t_{\mathrm{q}}$, as the elapsed time from having $\operatorname{EW}(\mathrm{H} \alpha)$ values consistent with those of a main sequence galaxy, to $\mathrm{EW}(\mathrm{H} \alpha) \sim 0$ (tracks in panel (a)), the values range from a few megayears for GDN8231 (i.e., SFR truncation) to $500 \mathrm{Myr}$ for GDN-17360. Interestingly, the SAM SFHs suggest that galaxies show pSB features as a result of fast quenching from the star-forming main sequence, without being preceded by a strong starburst (i.e., $\sim 3$ times higher SFR than the main sequence). A more practical definition of $t_{\mathrm{q}}$ as the elapsed time from $\log (\mathrm{sSFR} /$ $\left.\mathrm{Gyr}^{-1}\right)=0$ to -1 (tracks in panel (c)) results in similar values of $t_{\mathrm{q}}=300-800 \mathrm{Myr}$.
The fast-quenching scenario for all four galaxies agrees well with recent works that find strong Balmer absorptions on similarly young quiescent galaxies at $z \sim 1.5$ (Newman et al. 2010; Onodera et al. 2012; Bezanson et al. 2013; van de Sande et al. 2013; Belli et al. 2014a). It differs, however, with the results of Kriek et al. (2011), who studied a sample of stacked, high-resolution SEDs of galaxies at $0.5<z<2.0$, finding $\mathrm{EW}(\mathrm{H} \alpha)$ values consistent with gradually declining SFHs. Assuming the difference is not caused by the slightly different redshift range, a plausible explanation for this discrepancy is that there are indeed different evolutionary quenching tracks (e.g., Martin et al. 2007; Gonçalves et al. 2012; Schawinski et al. 2014 or Belli et al. 2015), and current spectroscopic samples at $z \gtrsim 1.5$ are still not fully representative of the whole quenching population.

Panel (d) of Figure 5 shows that the luminosity-weighted ages of the sample ranges from $t_{w}=700 \mathrm{Myr}$ for GDN-8231 to $t_{w}=1.1-1.3 \mathrm{Gyr}$ for the three quiescent galaxies. The SAM SFHs also suggest a formation redshift (i.e., onset of star formation) of $z_{\text {form }} \gtrsim 6$, a half-mass assembly by $z \sim 3$, and the onset of quenching by $z \sim 2$. Interestingly, the latter is similar to the $z_{\text {form }}$ of the galaxies inferred from $\tau$ models, which suggests a rapid build up $(\tau<100 \mathrm{Myr})$. This, however, is most likely a limitation of single-parameter $\tau$ models, which are biased toward short values of $\tau$ in order to reproduce the strong pSB features in the SED. Secular SFHs with rise and decay timescales of several hundred megayears appear to be more realistic, and are predicted by physically motivated models (e.g., Peng et al. 2010; Behroozi et al. 2013; Gladders et al. 2013). However, obtaining a direct, reliable measurement of the formation timescale would require additional measurements such as the metallicity $[\mathrm{Z} / \mathrm{H}]$ or the $\alpha$ element abundance $[\alpha / \mathrm{Fe}]$ which trace the evolution of the metals (e.g., Conroy 2013; Onodera et al. 2015).

The stars in panel (d) of Figure 5 show the age of the oldest, $t_{w}=1.5 \mathrm{Gyr}$, and youngest, $t_{w}=0.9 \mathrm{Gyr}$, quiescent galaxies 

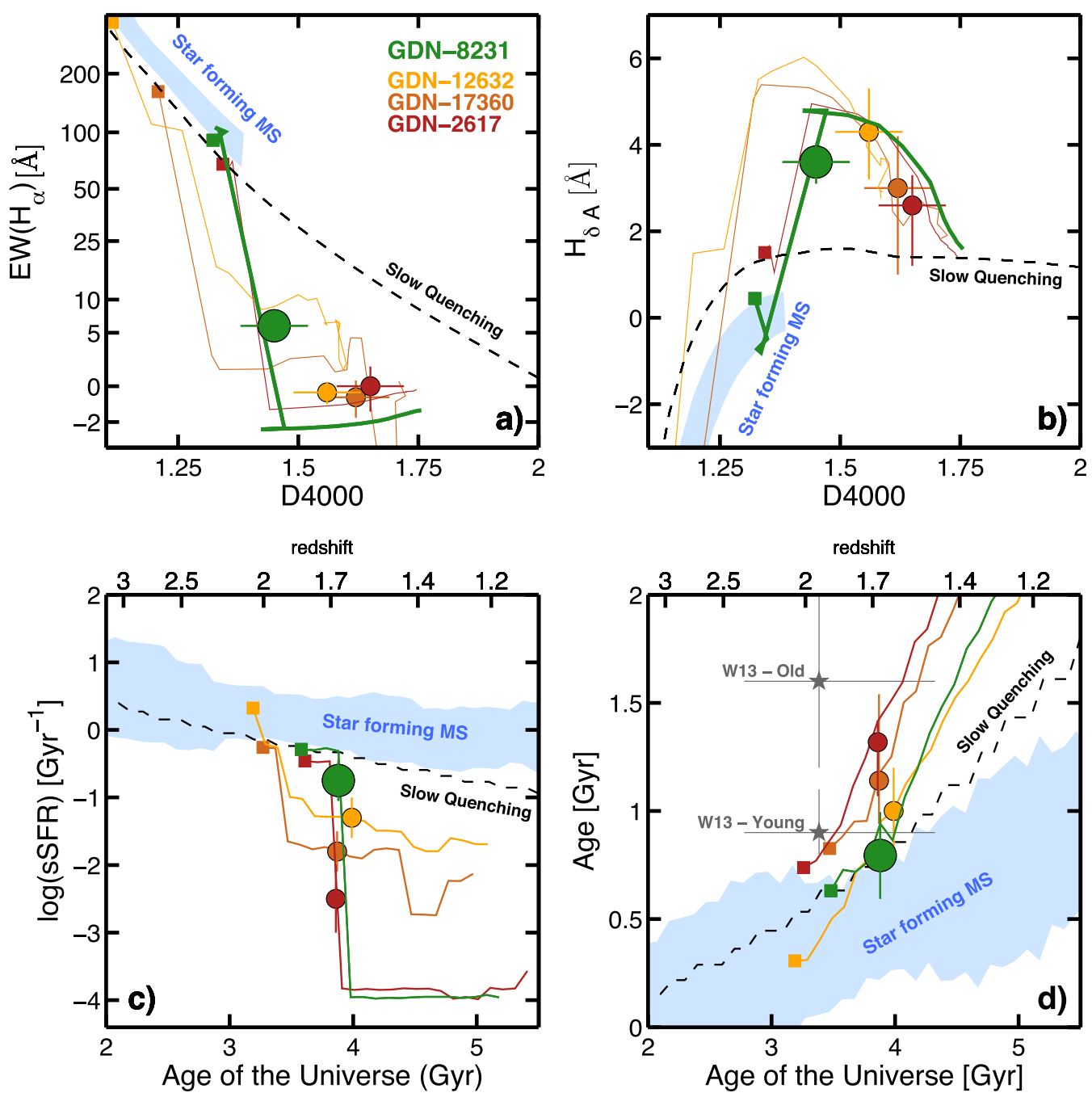

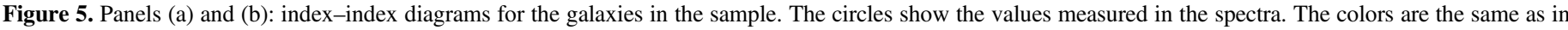

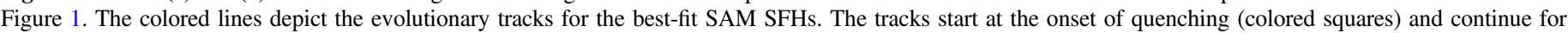

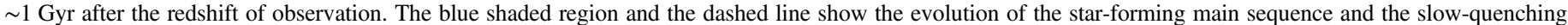

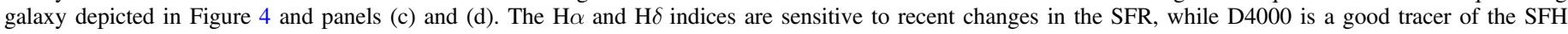

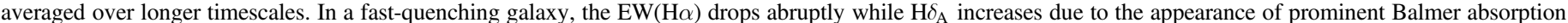

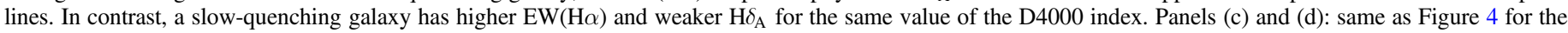

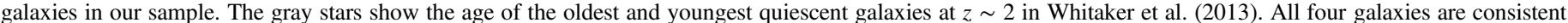

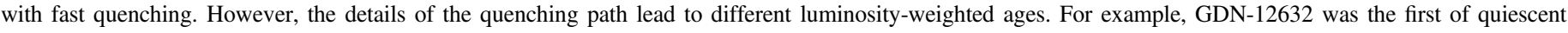
galaxies to quench and is the youngest, while GDN-2617 was the last to quench and it is the oldest.

at $z \sim 2$ from Whitaker et al. (2013) (see also Newman et al. 2015). The evolutionary tracks of the quiescent galaxies in our sample are consistent with being direct descendants of the youngest galaxies of Whitaker et al. (2013), and GDN-8231 reaches a similar age by $z=1.5$. This implies that as new quenching galaxies join the red sequence, the age spread in the quiescent population will increase by roughly 1 Gyr from $z=2$ to $z=1.5$. Interestingly, if all the galaxies in our sample follow a pure passive evolution since $z=1.7$, their ages by $z=0.6$ would be $t_{w} \sim 4.5-5 \mathrm{Gyr}$, which is roughly 2 Gyr older than the results of Choi et al. (2014) for the oldest quiescent galaxies at that redshift. This could be an indication that some quiescent galaxies retain low levels of star formation, or perhaps experience minor wet mergers that rejuvenate star formation.

In summary, the four galaxies in our sample have relatively young ages of $t_{w} \lesssim 1 \mathrm{Gyr}$ and present spectral features consistent with fast quenching of the star formation. In particular, GDN-8231 seems to be caught in an early stage of a rapid truncation.

\subsection{Kinematic Properties}

Figure 6 shows the $M_{\star}-\sigma_{\text {LOS }}$ relation for a compilation of compact SFGs from Barro et al. (2014b) and quiescent galaxies at $z \gtrsim 1.5$ from the literature (Bezanson et al. 2013; van de Sande et al. 2013; Belli et al. 2014a, 2014b). In Barro et al. (2014b), we found that (1) the gas kinematics of massive, compact SFGs are consistent with the stellar kinematics of compact quiescent galaxies, and (2) the dynamical masses of both populations are in good agreement with their stellar masses. We interpreted those similarities as evidence for an evolutionary connection. In particular, since both populations have similar structural, kinematic, and dynamical properties, compact SFGs simply turn into compact quiescent galaxies by quenching their star formation. In addition, one galaxy (COS- 


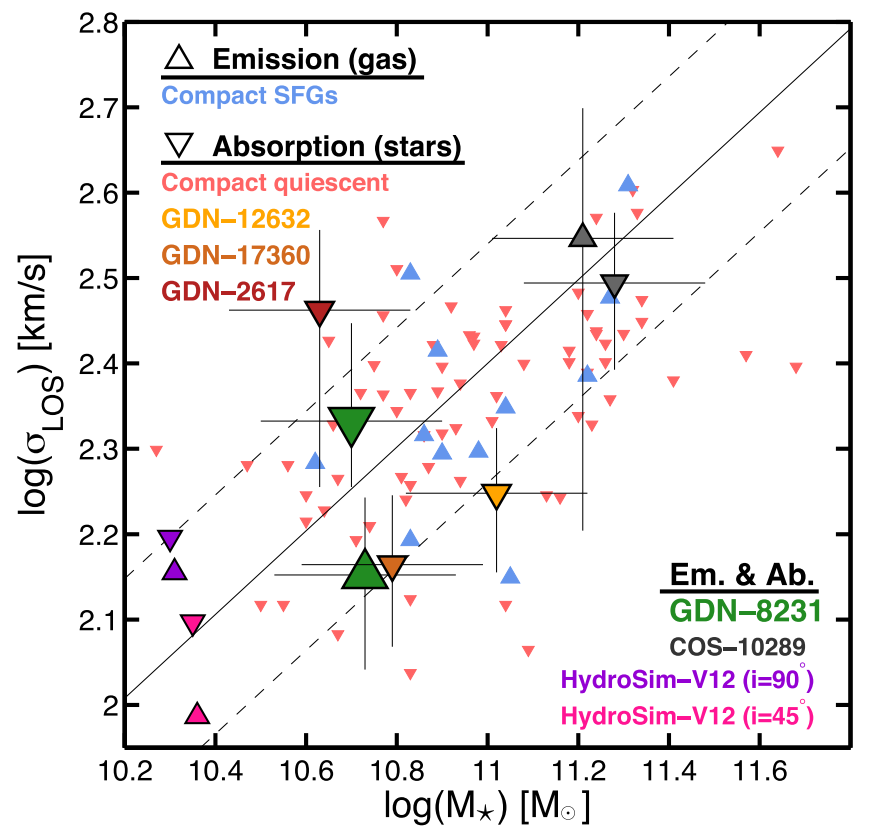

Figure 6. $\sigma_{\mathrm{LOS}}$ vs. $M_{\star}$ for different galaxy samples. The triangles pointing up and down show emission (gas) and absorption (stars) line measurements, respectively. The blue triangles depict 13 compact SFGs in Barro et al. (2014b) and 1 galaxy from Nelson et al. (2014). The red triangles show a compilation of quiescent galaxies (Bezanson et al. 2013; van de Sande et al. 2013; Belli et al. 2014a, 2014b) at $z \gtrsim 1.5$. The overlapping distributions for compact SFGs and quiescent galaxies suggests that both populations have similar kinematic properties. This is supported by the agreement in the gas and stellar dispersions of COS-10289 (Belli et al. 2014b, and Barro et al. 2014b for the stellar and gas kinematics). However, GDN-8231 (green) has $~ 40 \%$ lower dispersion in the gas than in the stars. A possible explanation is that the gas has colder kinematics than the stars $\left(v_{\phi} / \sigma_{r}>1\right)$, and thus its line-of-sight dispersion is prone to stronger projection effects. The gas and stellar dispersion for the simulated galaxy V12 (see also Figure 7) illustrates the bias toward lower values of $\sigma_{\text {LOS }}^{\text {gas }}$ with respect to $\sigma_{\text {LOS }}^{\star}$ for viewing angles closer to face-on (magenta).

10289) seemed to support this scenario by showing agreement between its gas and stellar kinematics. However, the low $\mathrm{S} / \mathrm{N}$ ratio of the emission line measurement and the presence of an $\mathrm{X}$-ray AGN within the galaxy place caveats on the interpretation of the kinematic properties of the gas.

Quite surprisingly, the better constrained measurements for GDN-8231 (green triangles in Figure 6) show that the gas has a lower velocity dispersion than the stars by a factor of $\sigma_{\text {LOS }}^{\star} / \sigma_{\text {LOS }}^{\text {gas }}=1.7 \pm 0.5$. Naively, we expected a value $\sim 1$ or, given that GDN-8231 is quenching, larger values of $\sigma_{\text {Los }}^{\text {gas }}$ caused by strong feedback processes (e.g., shocks or outflows; Diamond-Stanic et al. 2012; Genzel et al. 2014). In turn, the smaller values of $\sigma_{\mathrm{LOS}}^{\text {gas }}$ suggest that the gas is in dynamical equilibrium and that it has colder kinematic properties than the stars (i.e., higher $v_{\phi} / \sigma_{r}$ in the gas). If that is indeed the case, the lower dispersion in the gas could be the result of (1) widespread star-formation activity in a disk observed at low inclination (i.e., close to edge-on), or (2) a centrally concentrated star-forming region probing, on average, lower values of the rotational velocity $v_{\phi}$, which grows insideout. As described in Section 3.2, GDN-8231, has an inclination of $i=42^{\circ}$, and $20 \%$ smaller effective radius in the rest-frame NUV, which can lead to the smaller values of $\sigma_{\text {LOS }}^{\text {gas }}$ compared to $\sigma_{\mathrm{LOS}}^{\star}$.

In order to provide a better intuition, and quantify how much projection effects and/or a concentrated SFR profile affect the measurement of $\sigma_{\mathrm{LOS}}$, we study the kinematic profiles of the gas and the stars in V12, a high-resolution, hydrodynamic galaxy simulation ( $\sim 25 \mathrm{pc}$ grid) drawn from the sample of Zolotov et al. (2015) and Ceverino et al. (2014). As described in Zolotov et al. (2015), these galaxies have similar stellar and structural evolution as the compact SFGs and therefore, V12 provides a excellent proxy for the analysis of the kinematic properties of GDN-8231. Figure 7 shows that the stars in V12 have comparable rotation and dispersion, whereas the gas is rotation-dominated (stellar $v_{\phi} / \sigma_{r} \sim 1.4$ versus gas $v_{\phi} / \sigma_{r} \sim 5$ ). As a result, $\sigma_{\mathrm{LOS}}^{\text {gas }}$ is more sensitive to projection effects and shows smaller values for low inclinations. For example, the integrated, mass-weighted $\sigma_{\text {Los }}^{\text {gas }}$ at $r=r_{\mathrm{e}}$ show ratios of $\sigma_{\text {LOS }}^{\star} / \sigma_{\text {LOS }}^{\text {gas }} \sim 1.0$ and 1.5 for an edge-on $\left(i=90^{\circ}\right)$ and an intermediate inclination $\left(i=45^{\circ}\right)$, respectively (see values in Figure 6). Therefore, we conclude that a large fraction of the observed ratio of velocity dispersions in GDN-8231 can be accounted for by projection effects.

On the other hand, given the relatively flat $\sigma_{\text {LOS }}^{\text {gas }}$ profile of V12 (right panel of Figure 7), the change in the integrated value of $\sigma_{\text {LOS }}^{\text {gas }}\left(r_{\mathrm{e}}\right)$ would be small for a gas density $(\propto \mathrm{SFR})$ profile more centrally concentrated than the stellar profile (i.e., $\left.r_{\mathrm{e}, \star}>r_{\mathrm{e}, \text { gas }}\right)$. Using the ratio of effective radii in GDN-8231 $\left(r_{e, \mathrm{NUV}} / r_{e, V}=0.8\right)$ as an example of different gas-to-stellar concentrations, we find that integrating $\sigma_{\mathrm{LOS}}^{\text {gas }}$ only up $r=0.8 \mathrm{r}_{e}$ decreases its value by less than $5 \%$ for the edge-on case. Nevertheless, the effect of the gas density profile in $\sigma_{\text {LOS }}^{\text {gas }}$ can be larger in GDN-8231 if (1) it had a slowly increasing rotation curve and a flat $\sigma_{r}$ in the center, and/or (2) the emission line region, traced by $\mathrm{H} \alpha$, had an even smaller $r_{\mathrm{e}}$ than the NUV luminosity profile. The latter is more plausible given the quenching nature of GDN-8231 and the shorter star-formation timescales probed by $\mathrm{H} \alpha$.

Lastly, note that Figure 7 shows only the radial component of the intrinsic dispersion $\left(\sigma_{\mathrm{r}}\right)$ for V12. In the case of anisotropic dispersion, for example, due to strong collimated winds in the center of the galaxy $\left(\sigma_{\mathrm{z}} \gg \sigma_{\mathrm{r}}\right)$, the observed value of $\sigma_{\text {LOS }}^{\text {gas }}$ for a face-on inclination would be much larger than that of $\sigma_{\text {LOS }}^{\star}$.

\subsubsection{Dynamical Mass}

We estimate the dynamical mass of GDN-8231 from $\sigma_{\text {LOS }}^{\star}$, which, as described above, is less sensitive to projection effects. Following the virial equation

$$
M_{\mathrm{dyn}}\left(r<r_{e}\right)=K \frac{\sigma_{\mathrm{LOS}}^{2} r_{e}}{G}
$$

where $K$ depends on the galaxy's mass distribution, the inclination, and velocity field. We use $K=2.5$, which is the most widely adopted value for stars in dispersion dominated galaxies (e.g., Newman et al. 2010; van de Sande et al. 2013; Belli et al. 2014b), and is valid under a variety of galaxy geometries and mass distributions (e.g., Binney \& Tremaine 2008). We multiply this value by 2 to compare against the total mass (i.e., twice the mass enclosed within the halflight radius). We apply a small correction to scale the observed $\sigma_{\text {Los }}^{\star}$ to the value in a circular aperture of radius $r_{e}, \sigma_{e}=1.05 \times$ $\sigma_{\text {LoS }}^{\star}$ (Cappellari et al. 2006; van de Sande et al. 2011). The inferred dynamical mass $\log \left(M_{\text {dyn }} / M_{\odot}\right)=11.1$ is slightly larger than the stellar mass, but still consistent within the 

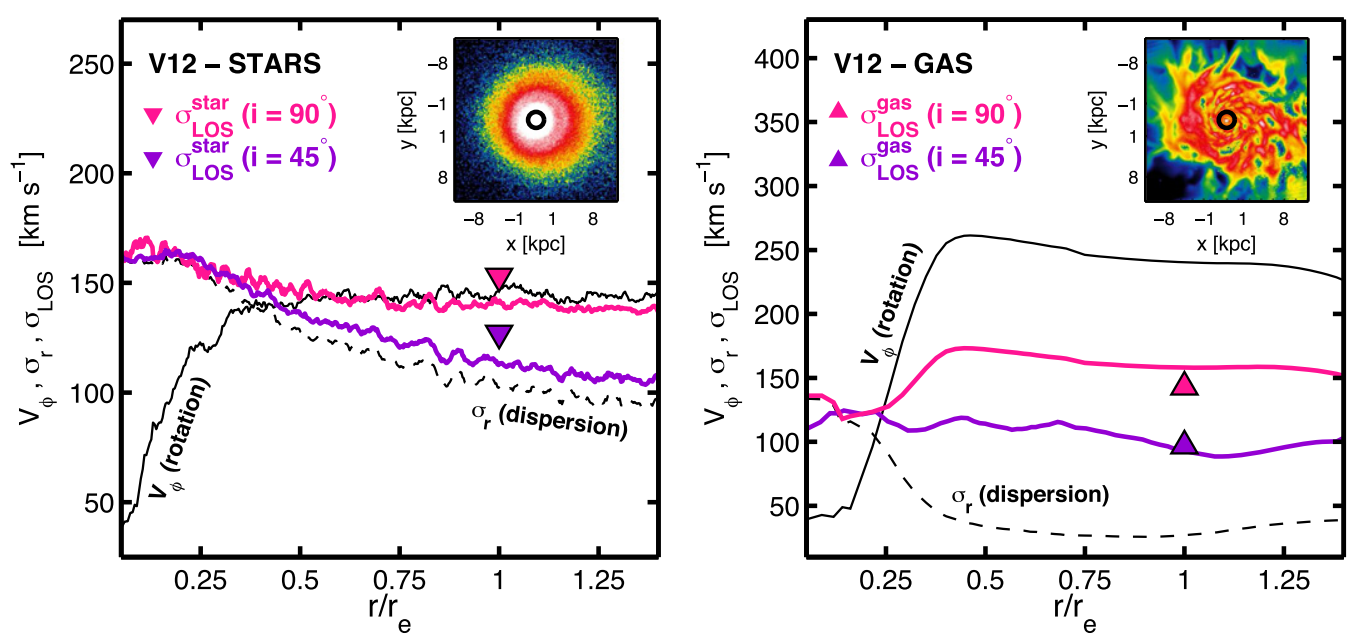

Figure 7. Stellar (left) and gas (right) kinematic profiles for the simulated galaxy V12 at $z=2.3$ (Ceverino et al. 2014; Zolotov et al. 2015). The kinematic properties are measured in cylindrical beams with a depth of $8 \mathrm{kpc}$. The solid and dashed black lines depict the intrinsic rotation and dispersion. The magenta and purple lines show the $\sigma_{\mathrm{LOS}}$ profiles for a line-of-sight inclination of $i=90^{\circ}$ (edge-on) and $i=45^{\circ}$. The triangles show the integrated, mass-weighted values at the $r=r_{\mathrm{e}}$. The lineof-sight dispersion can be written as $\sigma_{\mathrm{LOS}}^{2}=\beta\left(\sin i v_{\phi}\right)^{2}+\sigma_{r}^{2}$, where $\beta$ depends on the inclination and density profile of the galaxy. This implies that for a rotationdominated component the value of $\sigma_{\mathrm{LOS}}$ depends more strongly on projection effects. In V12 the gas has higher rotation than the stars $\left(v_{\phi} / \sigma_{r} \sim 1.4\right.$ vs. $\left.v_{\phi} / \sigma_{r} \sim 5\right)$ and therefore the ratio of velocity dispersions can be as high as $\sigma_{\mathrm{LOS}}^{\star} / \sigma_{\mathrm{LOS}}^{\mathrm{gas}} \sim 1.5$ for a LOS inclination of $i=45^{\circ}$. The insets in the upper right show the $10 \times 10 \mathrm{kpc}$ face-on density maps for the stars and the gas. The black circle has $r=r_{\mathrm{e}}$.

uncertainties in both the stellar mass and velocity dispersion. Previous studies of the $M_{\mathrm{dyn}} / M_{\star}$ ratio based on stellar velocity dispersion measurements in quiescent galaxies at $z \gtrsim 1.5$ find a similar offset toward larger dynamical masses by an average value of of $\log \left(M_{\mathrm{dyn}} / M_{\star}\right)=0.2 \mathrm{dex}$ for the most massive galaxies, $\log \left(M_{\mathrm{dyn}}\right)>11.2$, with a scatter of $\sim 0.15$ dex (Belli et al. 2014b). A similar comparison for sample of local quiescent galaxies drawn from the SDSS survey yields a similar offset and suggest that the difference increases with stellar mass as $M_{\text {dyn }}=0.15 M_{\star}^{1.09}$ (e.g., Bezanson et al. 2011; van de Sande et al. 2013) or with the distance of the galaxies to the quiescent mass-size relation (Peralta de Arriba et al. 2014). Note also that the emission lines in GDN-8231 suggest that there is still some gas remaining in the galaxy which contributes to the total baryonic mass $\left(M_{\text {baryon }}=M_{\star}+M_{\mathrm{gas}}\right)$ and therefore alleviates the tension in the comparison to the dynamical mass.

\section{CONCLUSIONS}

We present Keck I MOSFIRE NIR spectroscopy of GDN8231, a massive, compact SFG galaxy at $z \sim 1.7$. This galaxy was selected with sSFR and rest-frame colors matching an intermediate stage between star-forming and quiescent. The $Y$ and $H$-band spectra reveal strong Balmer absorption lines and $\mathrm{H} \alpha$ and $\left[\mathrm{N}_{\mathrm{II}}\right]$ in emission. The emission and absorption lines yield spectral indices and the kinematics of the gas and the stars.

The spectral indices, SED modeling, and the comparison to three compact quiescent galaxies at similar redshift indicate that GDN-8231 was caught in a rare early stage of fast quenching. Still relatively young, with a luminosity-weighted age of $700 \pm 250 \mathrm{Myr}$, GDN-8231 will mature to become a compact quiescent galaxy by redshift $z=1.5$. The rapid truncation of the SFR is evidenced by the low $\operatorname{EW}(\mathrm{H} \alpha)$ and weak MIPS $24 \mu \mathrm{m}$ flux. The color profile is bluer in the center, which is consistent with the predictions of recent simulations for an early stage of inside-out quenching. The line ratio of $[\mathrm{N}$ II] $/ \mathrm{H} \alpha \sim 1$ suggests the presence of a weak (not X-ray detected) AGN, a common finding among most compact SFGs (Barro et al. 2014a). Using SFHs based on SAMs, we find that the assembly of GDN-8231 is consistent with an early $(z \sim 6)$ onset of star formation, a secular build up in the star-forming main sequence, forming 50\% of its stellar mass before $z=3$, and fast quenching at $z \sim 1.7$. This is consistent with the notion that there is fast-quenching path at $z \sim 1.5$, as pointed out in previous works (Onodera et al. 2012; Bezanson et al. 2013). However, we caution that larger samples of quenching galaxies at this redshift are necessary to verify whether there is also a slow-quenching path.

In Barro et al. (2014b), we found that compact SFGs and quiescent galaxies have similar line-of-sight velocity dispersions for the gas and the stars, suggesting similar kinematics in progenitors and descendants. In GDN-8231, however, the dispersion of the gas, $\sigma_{\mathrm{LOS}}^{\text {gas }}=147 \pm 32 \mathrm{~km} \mathrm{~s}^{-1}$, is $40 \%$ smaller than that of the stars, $\sigma_{\text {Los }}^{\star}=215 \pm 35 \mathrm{~km} \mathrm{~s}^{-1}$. This difference can be explained if the gas has colder kinematics (rotationdominated) than the stars, and therefore (1) $\sigma_{\mathrm{LOS}}^{\text {gas }}$ is smaller if the viewing angle is low (close to face-on), and (2) $\sigma_{\text {Los }}^{\text {gas }}$ is smaller if the emission line (star-forming) region is concentrated at the center of the galaxy, and thus probes low values of $v_{\phi}$. These options are consistent with the findings of state-ofthe-art galaxy simulations which predict that the gas in compact SFGs reside in rotating disks (Zolotov et al. 2015). In the simulations, stars have $\sigma_{\text {tos }}^{\star}$ up to 1.5 times larger depending on the projection of the gas disk. A clear prediction of these models is that the compact quiescent descendants should retain some rotation from its disky progenitors.

GDN-8231 stresses the need for larger samples of compact SFGs with emission and absorption line kinematics to quantify the effects predicted in the simulations. Those samples would allow us to study the dependence of $\sigma_{\text {Los }}^{\star} / \sigma_{\text {Los }}^{\text {gas }}$ with the viewing angle and star formation activity. Similarly, high spatial resolution imaging in the sub-millimeter with ALMA, or in 

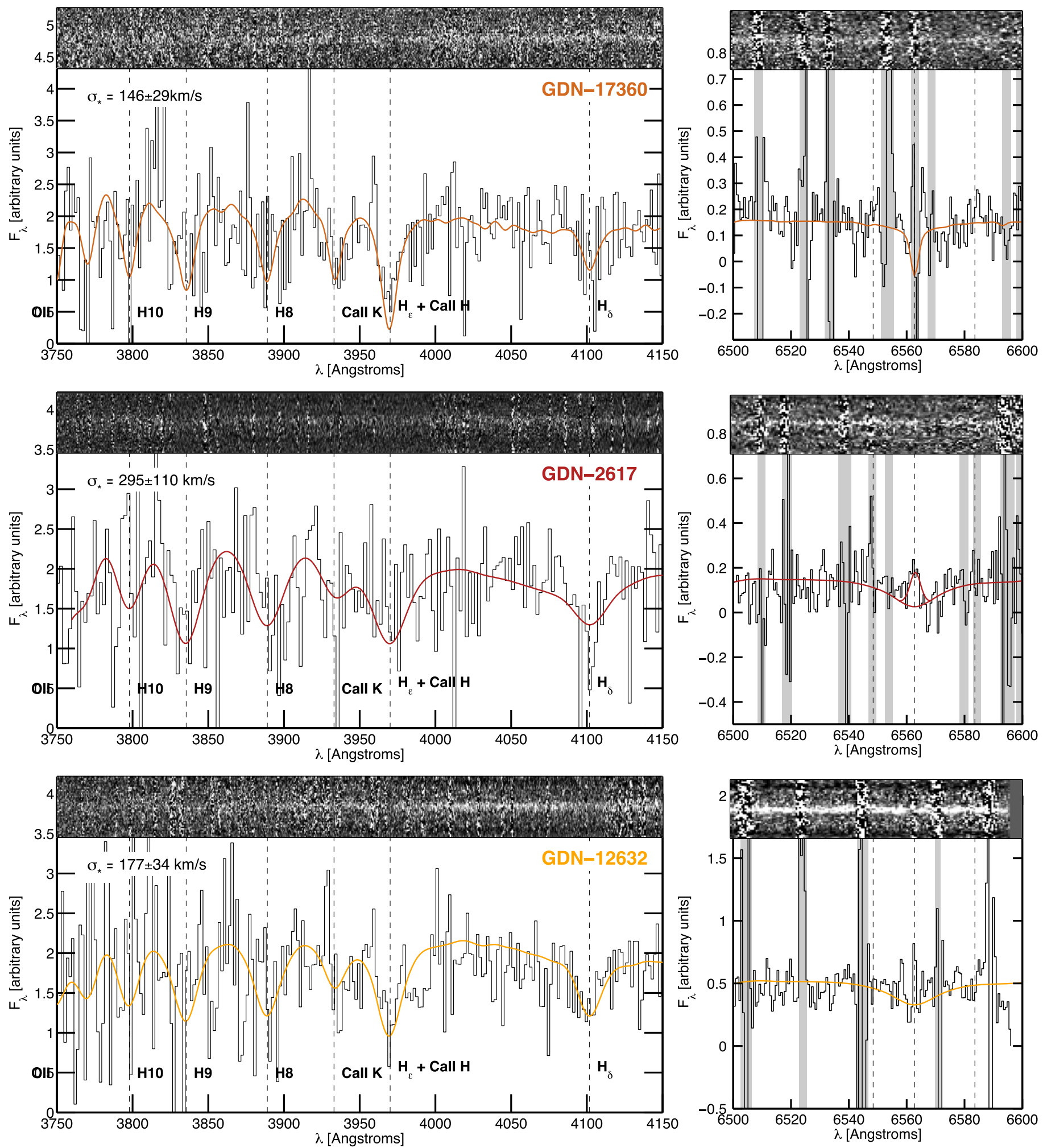

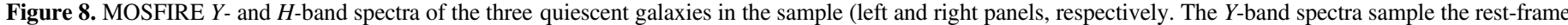

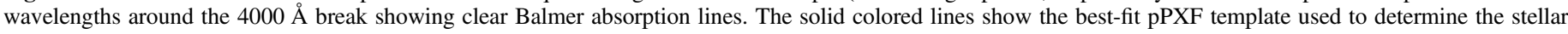

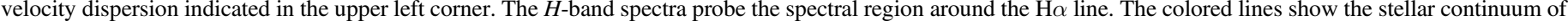

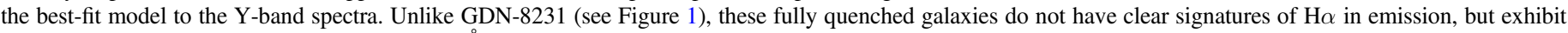
weak absorptions, consistent with $\mathrm{EW}(\mathrm{H} \alpha) \sim 0 \AA$, as expected from their overall older SEDs (see Figure 9).

the NIR with adaptive optics, can provide direct measurements of the size and location of the star-forming regions and, in some cases, resolved kinematics for the ionized gas.
Support for Program number HST-GO-12060 was provided by NASA through a grant from the Space Telescope Science Institute, which is operated by the Association of Universities 

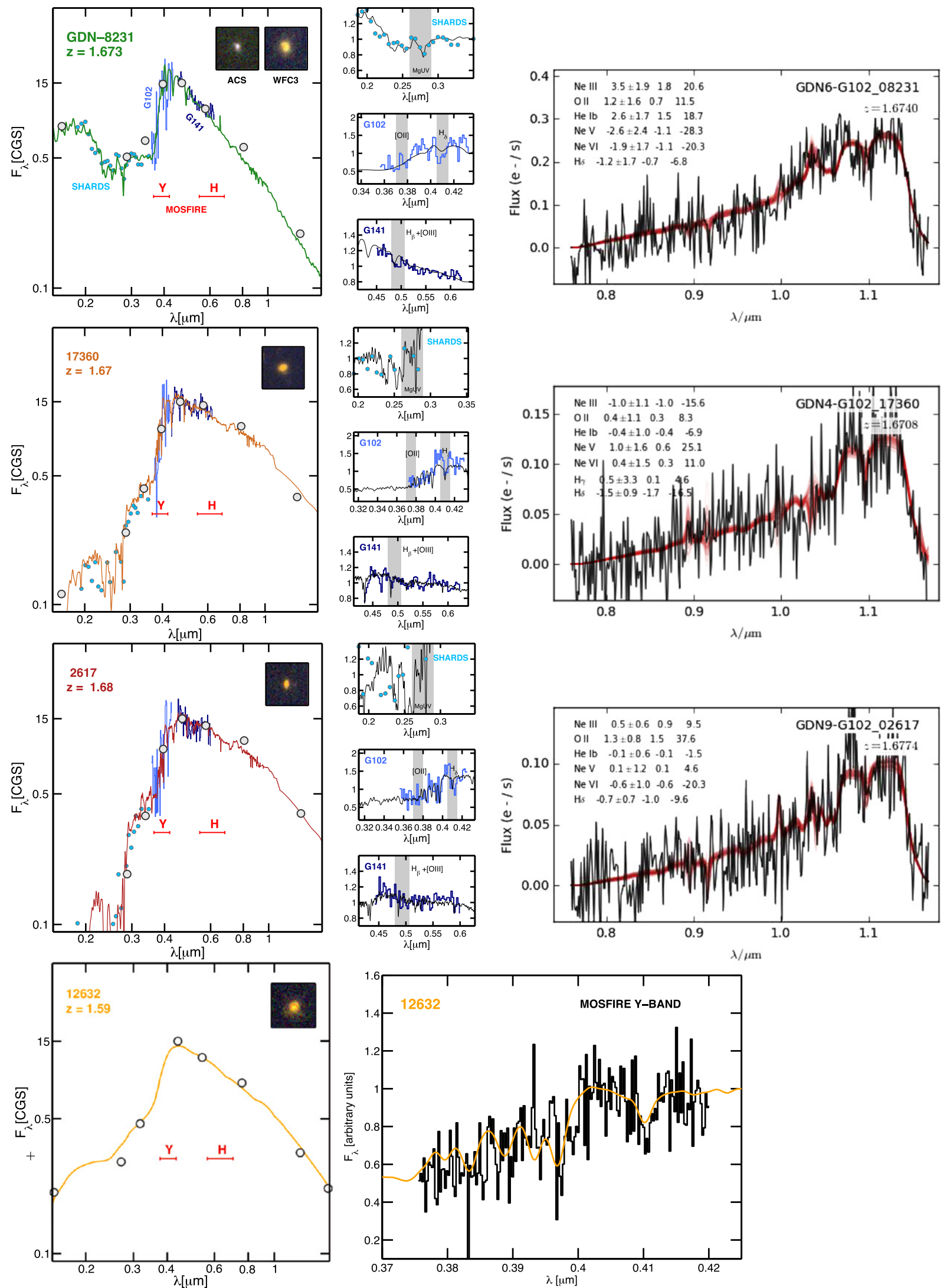

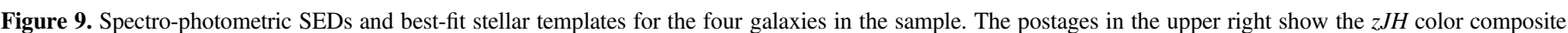

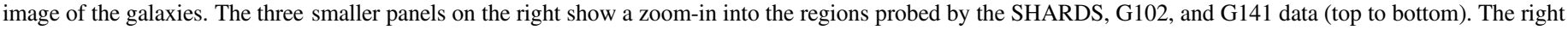

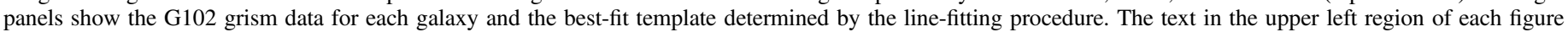

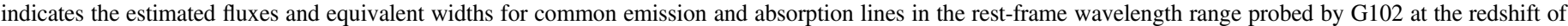
the galaxy. 

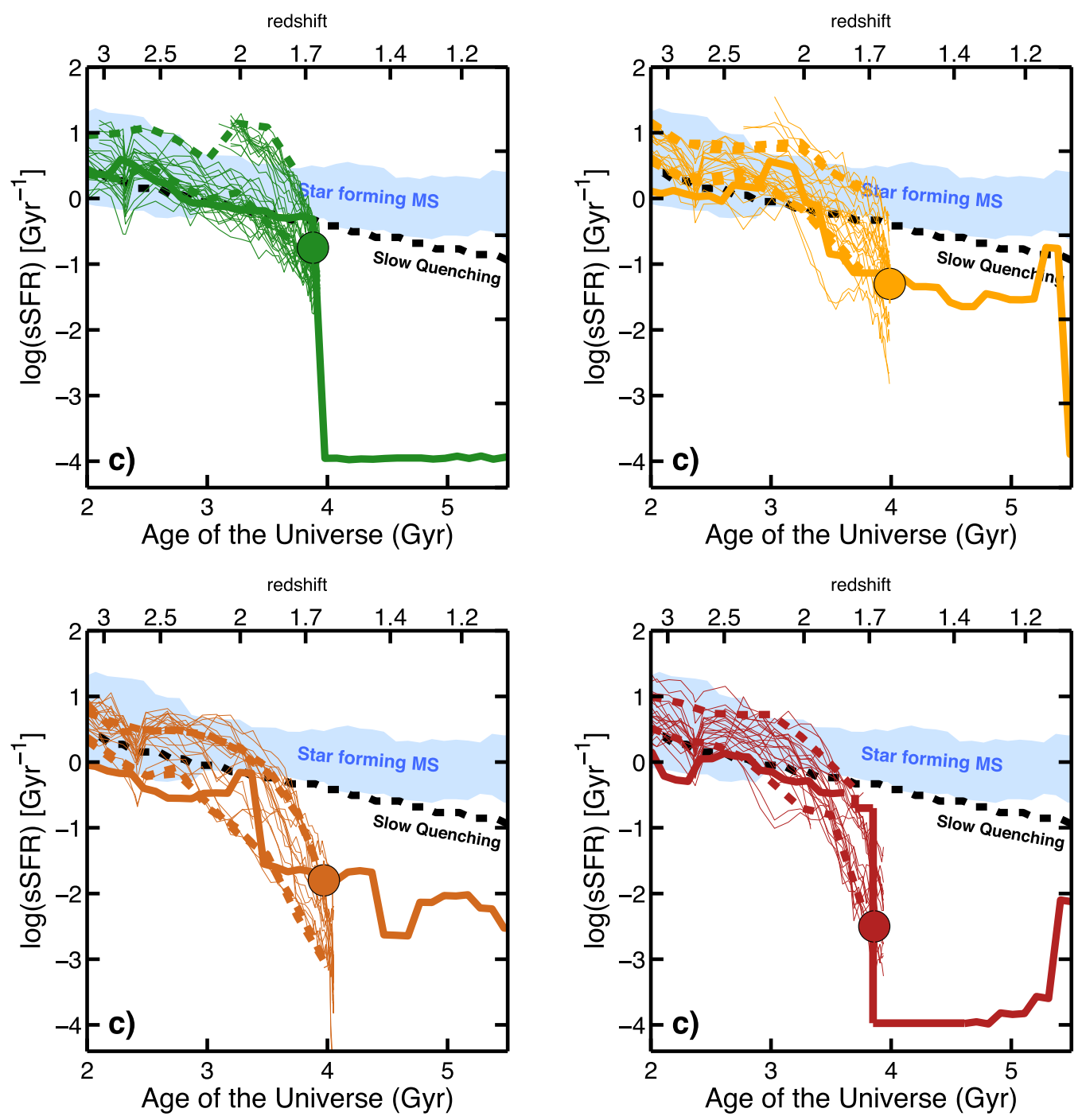

Figure 10. Evolutionary tracks in sSFR (left) vs. age of the universe for different SFHs for the galaxies in the sample. The blue shaded region and the dashed black line indicate the typical evolutionary tracks of a main sequence galaxy and a slow-quenching galaxy (see also Figure 4). The thick colored line in each panel shows the best-fit SFH (as in panel (c) of Figure 5). The thin lines depict all the SFHs consistent with the best fit within $3 \sigma$. The dashed lines show the upper and lower confidence ranges containing $95 \%$ of all possible SFHs computed in time-averaged intervals of $200 \mathrm{Myr}$.

for Research in Astronomy, Incorporated, under NASA contract NAS5-26555. G.B. acknowledges support from NSF grant AST-08-08133. P.G.P.-G. and M.C.E.M. acknowledge support from grant AYA2012-31277. J.R.T. acknowledges support from NASA through Hubble Fellowship grant \#51330. D.C. acknowledges support from AYA2012-32295. The simulations were performed at NASA Advanced Supercomputing (NAS) at NASA Ames Research Center. This work has made use of the Rainbow Cosmological Surveys Database, which is operated by the Universidad Complutense de Madrid (UCM), partnered with the University of California Observatories at Santa Cruz (UCO/Lick,UCSC). The authors recognize and acknowledge the very significant cultural role and reverence that the summit of Mauna Kea has always had within the indigenous Hawaiian community. We are most fortunate to have the opportunity to conduct observations from this mountain. Based partly on observations made with the Gran Telescopio Canarias (GTC), installed at the Spanish Observatorio del Roque de los Muchachos of the Instituto de Astrofsica de Canarias, in the island of La Palma.

\section{APPENDIX A \\ MOSFIRE SPECTRA AND SEDS OF THE THREE- QUIESCENT GALAXIES}

Figure 8 shows the $Y$ - and $H$-band spectra of the three quiescent galaxies in our sample following the same format as for GDN-8231 (Figure 2). The main difference between GDN-8231 and these quiescent galaxies is that the latter exhibit no clear traces of emission lines in the spectral range around $\mathrm{H} \alpha$ and [N II]. Only GDN-2617 (middle panel) has a tentative, weak $\mathrm{H} \alpha$ detection $(\mathrm{EW}(\mathrm{H} \alpha)<1.5 \AA)$. However, the $H$-band continuum in this galaxy has a low $\mathrm{S} / \mathrm{N}$ and thus the significance of the line is less than $2 \sigma$. Furthermore, unlike GDN-8231, the [N II] line is undetected. The $\mathrm{S} / \mathrm{N}$ per resolution element for the continuum of the galaxies in the $Y$ band spectra to the right of the $4000 \AA$ bright is slightly lower than in the spectrum of GDN-8231 ranging from $\mathrm{S} / \mathrm{N}=15$, 12 , and 7 in GDN-12632, GDN-17360, and GDN-2617. Based on these detections, we obtain velocity dispersion measurements with errors of the order of $20 \%$ for the first two, and $\sim 40 \%$ for GDN-2617. Note also that the $\mathrm{H} \delta_{\mathrm{A}}$ index used to 
constraint the $\mathrm{SFH}$ is measured on the red side of the spectrum where the $\mathrm{S} / \mathrm{N}$ is higher.

Figure 9 shows the composite broad-band, SHARDS, and grism SED of the galaxies in the sample jointly with the best-fit stellar template. The panels on the right depict the results of an independent estimate of the $\mathrm{H} \delta_{\mathrm{A}}$ absorption strength based on the G102 grism spectra. In spite of the lower spectral resolution of the HST grism, which depends on the spatial extent of the galaxy, these bright $(\mathrm{F} 105 \mathrm{~W}<22 \mathrm{mag})$ compact galaxies exhibit strong continuum detections in G102. The fit to absorption line is done with the software developed by the three-dimensional (3D) HST Team as a part of their G141 reduction and analysis pipeline (Momcheva et al. 2015). Briefly, the code directly fits the 2D spectrum using continuum and line templates. These line templates include multiple species that would be observed within the grism pass-band. The fit parameters are computed using a Monte Carlo Markov Chain method, emcee (Foreman-Mackey et al. 2013), to determine the marginalized posterior distribution functions. The spectra are normalized to the broad- and medium-band photometry (left panels of Figure 9). Given the lower resolution of the G102 spectra, we measure the $\mathrm{EW}(\mathrm{H} \delta)$ instead of $\mathrm{H} \delta_{\mathrm{A}}$ on the best-fit templates. The best templates and the confidence intervals from the Monte Carlo chains are shown as red lines in the right panels of Figure 9. The values of the rest-frame $\mathrm{EW}(\mathrm{H} \delta)$, given in Table 1, are consistent with the MOSFIRE measurements within the (larger) uncertainties. Galaxy GDN12632 lies outside the coverage of the G102 grism survey and thus such measurement is not possible. Nevertheless, this galaxy presents the highest $\mathrm{S} / \mathrm{N}$ continuum detection in the MOSFIRE $Y$-band spectrum. The bottom panel of Figure 9 shows the overall SED and the $Y$-band spectrum of this galaxy used to compute both $\mathrm{H} \delta_{\mathrm{A}}$ and the D4000 indices. Nonetheless, these are very similar differing only in the definition of the continuum bands

\section{APPENDIX B}

\section{THE RANGE OF POSSIBLE SFHs FOR THE GALAXIES}

Figure 10 shows the sSFR versus age diagram for the four galaxies in the sample following the same nomenclature as in panel (c) of Figure 5. The goal of this figure is to illustrate the range of possible SFHs that are consistent with the observed SEDs of the four galaxies in our sample. The solid lines show best-fit SFHs (same as in Figure 5), while the dashed lines depict the $95 \%$ upper and lower limits of the sSFR relative to the average $\mathrm{SFH}$. These are determined from a subset of the SFHs in the fitting library that have a $\chi^{2}$ within $3 \sigma$ of the best model. Note that confidence intervals are not strictly a SFH, but rather averages of individual SFH in bins of time.

The range of possible solutions illustrates that while the SED fit provides relatively wide constraints on the overall SFH (see also Pacifici et al. 2015). By using spectral indices sensitive to the recent star formation as priors $\left(\mathrm{EW}(\mathrm{H} \alpha)\right.$ and $\left.\mathrm{H} \delta_{\mathrm{A}}\right)$, we obtain tighter constraints on the quenching path of the galaxy. In particular, all these galaxies must exhibit a recent truncation of the SFR (deviating from the blue region and black dashed line) in order for their SEDs to show the characteristic spectral features of a post-starburst galaxy shown in the index-index diagrams of panels (a) and (b) of Figure 5.

Our inferences based on the comparison of the spectral indices to the evolutionary tracks from SFHs in the indexindex diagrams (Figure 5) are very similar to previous results presented in studies of rapidly quenching, post-starburst galaxies (e.g, Wild et al. 2009; Figure 6). The main addition is that in this analysis we use a library of physically motivated SFHs to constrain the most likely evolution from the fit to both the spectral indices and the overall SED, rather than comparing the indices to a grid of parametric SFH models, typically SSPs or exponentially declining, with different quenching times ranging from a few megayears to a few hundred megayears (e.g., Marcillac et al. 2006; Onodera et al. 2012).

\section{REFERENCES}

Alexander, D. M., Bauer, F. E., Brandt, W. N., et al. 2003, AJ, 126, 539 Baldwin, J. A., Phillips, M. M., \& Terlevich, R. 1981, PASP, 93, 5 Balogh, M. L., Morris, S. L., Yee, H. K. C., Carlberg, R. G., \& Ellingson, E. 1999, ApJ, 527, 54

Barro, G., Faber, S. M., Pérez-González, P. G., et al. 2013, ApJ, 765, 104 Barro, G., Faber, S. M., Pérez-González, P. G., et al. 2014a, ApJ, 791, 52 Barro, G., Trump, J. R., Koo, D. C., et al. 2014b, ApJ, 795, 145 Bedregal, A. G., Scarlata, C., Henry, A. L., et al. 2013, ApJ, 778, 126 Behroozi, P. S., Wechsler, R. H., \& Conroy, C. 2013, ApJ, 770, 57 Belli, S., Newman, A. B., \& Ellis, R. S. 2014a, ApJ, 783, 117 Belli, S., Newman, A. B., \& Ellis, R. S. 2015, ApJ, 799, 206 Belli, S., Newman, A. B., Ellis, R. S., \& Konidaris, N. P. 2014b, ApJL, 788, L29

Bezanson, R., van Dokkum, P., van de Sande, J., Franx, M., \& Kriek, M. 2013, ApJL, 764, L8

Bezanson, R., van Dokkum, P. G., Franx, M., et al. 2011, ApJL, 737, L31

Binney, J., \& Tremaine, S. 2008, Galactic Dynamics (2nd ed.; Princeton, NJ: Princeton Univ. Press)

Brammer, G. B., van Dokkum, P. G., \& Coppi, P. 2008, ApJ, 686, 1503

Brammer, G. B., van Dokkum, P. G., Franx, M., et al. 2012, ApJS, 200, 13

Bruzual, G., \& Charlot, S. 2003, MNRAS, 344, 1000

Buitrago, F., Trujillo, I., Conselice, C. J., et al. 2008, ApJ, 687, L61

Calzetti, D., Armus, L., Bohlin, R. C., et al. 2000, ApJ, 533, 682

Cappellari, M., Bacon, R., Bureau, M., et al. 2006, MNRAS, 366, 1126

Cappellari, M., \& Emsellem, E. 2004, PASP, 116, 138

Carollo, C. M., Bschorr, T. J., Renzini, A., et al. 2013, ApJ, 773, 112

Cassata, P., Giavalisco, M., Guo, Y., et al. 2011, ApJ, 743, 96

Cenarro, A. J., Peletier, R. F., Sánchez-Blázquez, P., et al. 2007, MNRAS, 374,664

Ceverino, D., Dekel, A., \& Bournaud, F. 2010, MNRAS, 404, 2151

Ceverino, D., Klypin, A., Klimek, E. S., et al. 2014, MNRAS, 442, 1545

Chabrier, G. 2003, PASP, 115, 763

Cheung, E., Faber, S. M., Koo, D. C., et al. 2012, ApJ, 760, 131

Choi, J., Conroy, C., Moustakas, J., et al. 2014, ApJ, 792, 95

Cimatti, A., Cassata, P., Pozzetti, L., et al. 2008, A\&A, 482, 21

Conroy, C. 2013, ARA\&A, 51, 393

Conroy, C., \& Gunn, J. E. 2010, ApJ, 712, 833

Courteau, S., Dutton, A. A., van den Bosch, F. C., et al. 2007, ApJ, 671, 203

Daddi, E., Renzini, A., Pirzkal, N., et al. 2005, ApJ, 626, 680

Dale, D. A., Giovanelli, R., Haynes, M. P., et al. 1997, AJ, 114, 455

Damjanov, I., McCarthy, P. J., Abraham, R. G., et al. 2009, ApJ, 695, 101

Dekel, A., Birnboim, Y., Engel, G., et al. 2009, Natur, 457, 451

Dekel, A., \& Burkert, A. 2014, MNRAS, 438, 1870

Dekel, A., Zolotov, A., Tweed, D., et al. 2013, MNRAS, 435, 999

Diamond-Stanic, A. M., Moustakas, J., Tremonti, C. A., et al. 2012, ApJL, 755, L26

Elbaz, D., Daddi, E., Le Borgne, D., et al. 2007, A\&A, 468, 33

Elbaz, D., Dickinson, M., Hwang, H. S., et al. 2011, A\&A, 533, A119

Falcón-Barroso, J., Sánchez-Blázquez, P., Vazdekis, A., et al. 2011, A\&A, 532, A95

Fang, J. J., Faber, S. M., Koo, D. C., \& Dekel, A. 2013, ApJ, 776, 63

Foreman-Mackey, D., Hogg, D. W., Lang, D., \& Goodman, J. 2013, PASP, 125,306

Galametz, A., Grazian, A., Fontana, A., et al. 2013, ApJS, 206, 10

Genzel, R., Förster Schreiber, N. M., Rosario, D., et al. 2014, ApJ, 796, 7

Gladders, M. D., Oemler, A., Dressler, A., et al. 2013, ApJ, 770, 64

Gonçalves, T. S., Martin, D. C., Menéndez-Delmestre, K., Wyder, T. K., \& Koekemoer, A. 2012, ApJ, 759, 67

Grogin, N. A., Kocevski, D. D., Faber, S. M., et al. 2011, ApJS, 197, 35

Guo, Y., Ferguson, H. C., Giavalisco, M., et al. 2013, ApJS, 207, 24

Guo, Y., Giavalisco, M., Cassata, P., et al. 2012, ApJ, 749, 149

Haynes, M. P., \& Giovanelli, R. 1984, AJ, 89, 758 
Hernán-Caballero, A., Alonso-Herrero, A., Pérez-González, P. G., et al. 2014, MNRAS, 443, 3538

Hopkins, P. F., Hernquist, L., Cox, T. J., et al. 2006, ApJS, 163, 1

Kauffmann, G., Heckman, T. M., Tremonti, C., et al. 2003, MNRAS, 346, 1055

Kelson, D. D., Illingworth, G. D., Franx, M., \& van Dokkum, P. G. 2001, ApJL, 552, L17

Kennicutt, R. C., Jr. 1998, ARA\&A, 36, 189

Koekemoer, A. M., Faber, S. M., Ferguson, H. C., et al. 2011, ApJS, 197, 36

Kriek, M., Shapley, A. E., Reddy, N. A., et al. 2015, ApJS, 218, 15

Kriek, M., van Dokkum, P. G., Labbé, I., et al. 2009, ApJ, 700, 221

Kriek, M., van Dokkum, P. G., Whitaker, K. E., et al. 2011, ApJ, 743, 168

Liu, F. S., Guo, Y., Koo, D. C., et al. 2013, ApJ, 769, 147

Magdis, G. E., Rigopoulou, D., Huang, J.-S., \& Fazio, G. G. 2010, MNRAS, 401, 1521

Marcillac, D., Elbaz, D., Charlot, S., et al. 2006, A\&A, 458, 369

Martin, D. C., Wyder, T. K., Schiminovich, D., et al. 2007, ApJS, 173, 342

McLean, I. S., Steidel, C. C., Epps, H., et al. 2010, Proc. SPIE, 7735, 77351E

McLean, I. S., Steidel, C. C., Epps, H. W., et al. 2012, Proc. SPIE, 8446, 84460J

Miller, S. H., Bundy, K., Sullivan, M., Ellis, R. S., \& Treu, T. 2011, ApJ, 741,115

Momcheva, I. G., Brammer, G. B., van Dokkum, P. G., et al. 2015, e-print (arXiv:1510.02106)

Muzzin, A., Marchesini, D., van Dokkum, P. G., et al. 2009, ApJ, 701, 1839

Naab, T., Johansson, P. H., Ostriker, J. P., \& Efstathiou, G. 2007, ApJ, 658,710

Nelson, E., van Dokkum, P., Franx, M., et al. 2014, Natur, 513, 394

Newman, A. B., Belli, S., \& Ellis, R. S. 2015, ApJL, 813, L7

Newman, A. B., Ellis, R. S., Bundy, K., \& Treu, T. 2012a, ApJ, 746, 162

Newman, A. B., Ellis, R. S., Treu, T., \& Bundy, K. 2010, ApJL, 717, L103

Newman, S. F., Genzel, R., Förster-Schreiber, N. M., et al. 2012b, ApJ, 761, 43

Noeske, K. G., Weiner, B. J., Faber, S. M., et al. 2007, ApJL, 660, L43

Onodera, M., Carollo, C. M., Renzini, A., et al. 2015, ApJ, 808, 161

Onodera, M., Renzini, A., Carollo, M., et al. 2012, ApJ, 755, 26

Pacifici, C., Charlot, S., Blaizot, J., \& Brinchmann, J. 2012, MNRAS, 421, 2002

Pacifici, C., da Cunha, E., Charlot, S., et al. 2015a, MNRAS, 447, 786

Pannella, M., Carilli, C. L., Daddi, E., et al. 2009, ApJL, 698, L116

Pannella, M., Elbaz, D., Daddi, E., et al. 2015, ApJ, 807, 141

Patel, S. G., van Dokkum, P. G., Franx, M., et al. 2013, ApJ, 766, 15

Peng, Y.-j., Lilly, S. J., Kovač, K., et al. 2010, ApJ, 721, 193

Peralta de Arriba, L., Balcells, M., Falcón-Barroso, J., \& Trujillo, I. 2014 MNRAS, 440, 1634

Pérez-González, P. G., Cava, A., Barro, G., et al. 2013, ApJ, 762, 46

Price, S. H., Kriek, M., Brammer, G. B., et al. 2014, ApJ, 788, 86
Prugniel, P., Soubiran, C., Koleva, M., \& Le Borgne, D. 2007, arXiv: 1102.2736

Rodighiero, G., Cimatti, A., Gruppioni, C., et al. 2010, A\&A, 518, L25

Salim, S., Rich, R. M., Charlot, S., et al. 2007, ApJS, 173, 267

Sánchez-Blázquez, P., Gorgas, J., Cardiel, N., \& González, J. J. 2006a, A\&A, 457, 787

Sánchez-Blázquez, P., Peletier, R. F., Jiménez-Vicente, J., et al. 2006b, MNRAS, 371, 703

Schawinski, K., Urry, C. M., Simmons, B. D., et al. 2014, MNRAS, 440, 889

Stefanon, M., Marchesini, D., Rudnick, G. H., Brammer, G. B., \& Whitaker, K. E. 2013, ApJ, 768, 92

Szomoru, D., Franx, M., \& van Dokkum, P. G. 2012, ApJ, 749, 121

Toft, S., Gallazzi, A., Zirm, A., et al. 2012, ApJ, 754, 3

Trager, S. C., Faber, S. M., Worthey, G., \& González, J. J. 2000, AJ, 119, 1645

Tran, K.-V. H., Nanayakkara, T., Yuan, T., et al. 2015, ApJ, 811, 28

Trujillo, I., Conselice, C. J., Bundy, K., et al. 2007, MNRAS, 382, 109

Trump, J. R., Konidaris, N. P., Barro, G., et al. 2013, ApJL, 763, L6

Valdes, F., Gupta, R., Rose, J. A., Singh, H. P., \& Bell, D. J. 2004, ApJS, 152,251

van de Sande, J., Kriek, M., Franx, M., et al. 2011, ApJL, 736, L9

van de Sande, J., Kriek, M., Franx, M., et al. 2013, ApJ, 771, 85

van der Wel, A., Franx, M., van Dokkum, P. G., et al. 2014, ApJ, 788, 28

van Dokkum, P. G., Bezanson, R., van der Wel, A., et al. 2014, ApJ, 791, 45

van Dokkum, P. G., \& Brammer, G. 2010, ApJL, 718, L73

van Dokkum, P. G., Franx, M., Kriek, M., et al. 2008, ApJL, 677, L5

Wellons, S., Torrey, P., Ma, C.-P., et al. 2015, MNRAS, 449, 361

Whitaker, K. E., Franx, M., Leja, J., et al. 2014, ApJ, 795, 104

Whitaker, K. E., Kriek, M., van Dokkum, P. G., et al. 2012a, ApJ, 745, 179

Whitaker, K. E., Labbé, I., van Dokkum, P. G., et al. 2011, ApJ, 735, 86

Whitaker, K. E., van Dokkum, P. G., Brammer, G., \& Franx, M. 2012b, ApJL, 754, L29

Wild, V., Almaini, O., Cirasuolo, M., et al. 2014, MNRAS, 440, 1880

Wild, V., Heckman, T., \& Charlot, S. 2010, MNRAS, 405, 933

Wild, V., Walcher, C. J., Johansson, P. H., et al. 2009, MNRAS, 395, 144

Williams, R. J., Quadri, R. F., Franx, M., et al. 2010, ApJ, 713, 738

Worthey, G., \& Ottaviani, D. L. 1997, ApJS, 111, 377

Wuyts, S., Cox, T. J., Hayward, C. C., et al. 2010, ApJ, 722, 1666

Wuyts, S., Förster Schreiber, N. M., Genzel, R., et al. 2012, ApJ, 753, 114

Wuyts, S., Förster Schreiber, N. M., Lutz, D., et al. 2011a, ApJ, 738, 106

Wuyts, S., Förster Schreiber, N. M., van der Wel, A., et al. 2011b, ApJ, 742, 96

Wuyts, S., Labbé, I., Franx, M., et al. 2007, ApJ, 655, 51

Yan, R., Newman, J. A., Faber, S. M., et al. 2006, ApJ, 648, 281

Yesuf, H. M., Faber, S. M., Trump, J. R., et al. 2014, ApJ, 792, 84

Zolotov, A., Dekel, A., Mandelker, N., et al. 2015, MNRAS, 450, 2327 